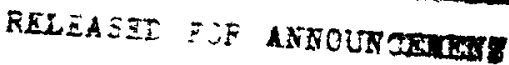

IF DUCELAF SEIENCE AgETRACTS

UNIVERSITY OF CALIFORNIA

Lawrence Radiation Laboratory

Livermore, California

AEC Contract No. W-7405-eng-48

\section{CESTI PRICAS}

н. $\$ 3,02$. 12

\title{
LECTURES ON HYDRODYNAMICS AND SHOCK WAVES
}

Lectures by Robert Lelevier

Notes by Boris Ragent

September 17, 1965

\section{LEGAL NOTICE} was prepared as an account acting on behalf of the Commission the accuTh1s report was prepasion, nor any person acting onsed or implied, uith respect to the ace use States, nor the Cow warranty or representation, emation contained in this report, oy not infringe A Makes any warrasefulness of the informates disclosed in this report may not me racy, completentis, apparatus, method. or proces an infor or famages resul privately owned rights, or B. Assumes any liabsilu mas, method, or process dis commission" includes any that se of any information, appar "person acting on behall of then contractor, to the oxtent thes, us used in the above, Commission, or employee of such of such contractor prepares, pr contractor of the Com the Commission, of employt to his employment or contract ployee er cloyee or contractor of the Cany information pursuant disseminates, or provides access to, anent with such contractor. with the Commission, or his employment w 


\section{DISCLAIMER}

This report was prepared as an account of work sponsored by an agency of the United States Government. Neither the United States Government nor any agency Thereof, nor any of their employees, makes any warranty, express or implied, or assumes any legal liability or responsibility for the accuracy, completeness, or usefulness of any information, apparatus, product, or process disclosed, or represents that its use would not infringe privately owned rights. Reference herein to any specific commercial product, process, or service by trade name, trademark, manufacturer, or otherwise does not necessarily constitute or imply its endorsement, recommendation, or favoring by the United States Government or any agency thereof. The views and opinions of authors expressed herein do not necessarily state or reflect those of the United States Government or any agency thereof. 


\section{DISCLAIMER}

Portions of this document may be illegible in electronic image products. Images are produced from the best available original document. 


\title{
LECTURES ON HYDRODYNAMICS AND SHOCK WAVES
}

Lectures by Robert Lelevier

Notes by Boris Ragent

Lawrence Radiation Laboratory, University of California

Livermore, California

September 17, 1965

\begin{abstract}
The purpose of these notes is to present an elementary treatment of shock hydrodynamics in a convenient form. Following a brief formulation of the mathematical description of continuous flow, three main topics are discussed. These are (1) the Hugoniot relations for shocks, (2) the method of characteristics as applied to one-dimensional gases and (3) methods of integration using high-speed digital computers.

It is hoped that these notes will provide a basis for understanding the work in this field, particularly for those groups not directly concerned with problems in shock hydrodynamics.
\end{abstract}

\section{INTRODUCTION}

\section{References}

Sir Horace Lamb, Hydrodynamics, 6th ed. N. Y., Dover, 1945. (Note: The Rayleigh approach to continuous flow - classical and slightly out of date.)

Richard Courant and K. O. Friedrichs, Supersonic Flow and Shock Waves, N.Y., Interscience, 1948.

Ascher H. Shapiro, The Dynamics and Thermodynamics of Compressible Fluid Flow, N. Y., Ronald press, c 1953.

Walker Bleakney and A. H. Taub, "Interaction of Shock Waves," Reviews of Mod. Phys., 21, 584-605 (1949). (Note: Elegant derivation of the equations of motion and the formation of shock. Good article.)

Outline of General Program

This series of lectures will deal with the following general topics:

1. Continuous hydrodynamics.

2. Shock hydrod, namics.

3. Numerical and analytical methods. 


\section{Preliminary Discussion}

In this discussion we will deal with macroscopic descriptions of a fluid, ignoring the questions of the microscopic properties of the particles of the fluid. Therefore, we restrict our attention to regions for which the mean free path for particles of the fluid is small compared with the dimensions of the system under consideration. The results derived from such considerations are in good agreement with experiment. Phenomena involving viscosity and heat conduction will be ignored. That is, we shall consider problems in which the energy transport by these processes is small compared to that involved in the hydrodynamic flow. Even with this simplification only a small class of problems can be solved analytically. In most cases one relies on a numerical integration performed on high speed digital computers.

\section{DEFINITIONS}

1. Fluid: A substance that deforms continuously under shearing forces.

2. Properties of a fluid:
a. Density
b. Velocity of flow
c. Stress in the fluid or "pressure."

The pressure of a fluid is defined as follows. Consider a small element of surface area $\Delta \mathrm{A}$ with surface normal $\vec{n}$, as in Fig. 1 . Then

$$
\begin{aligned}
& \text { pressure }=\lim _{\Delta A \rightarrow 0} \frac{\vec{F} \cdot \vec{n}}{\Delta A} ; \\
& \text { where in } \Delta A \rightarrow 0 \text { we keep in }
\end{aligned}
$$
mind the macroscopic nature of our considerations.

The pressure is a scalar quantity.

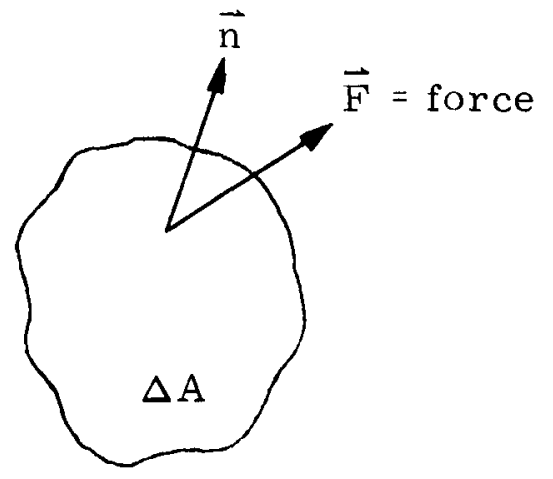

Fig. 1 
Proof:

Consider a two-dimensional

fluid and a triangular fluid element of sides $d x, d y, \sqrt{d x^{2}+d y^{2}}$, and density $\rho$ oriented as shown in Fig. 2. Let $g$ be a gravitational acceleration, $m$ the mass, $a$ the acceleration, and $\gamma$ the angle as shown in Fig. 2. The forces acting at point $P$ in the $x$ direction in the triangle are, from Newton's law

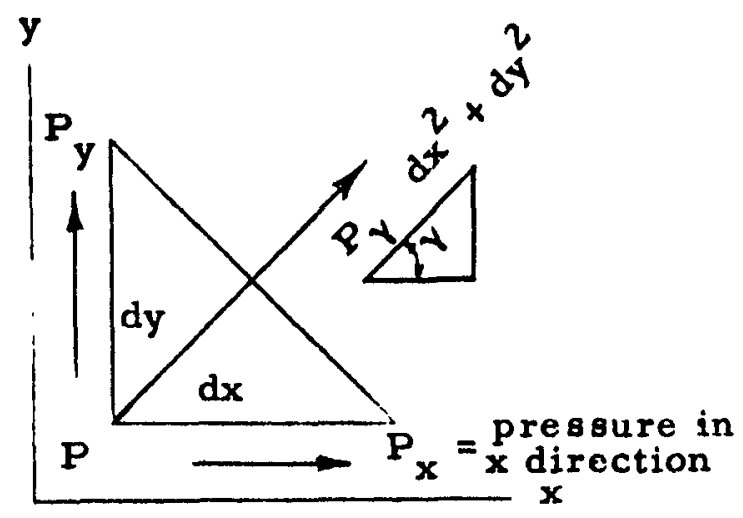

Fig. 2

$\Sigma F_{x}=\operatorname{ma}_{x}$, or $\left(P_{x} d y \cdot 1\right)-P_{\gamma} \sqrt{d x^{2}+d y^{2}} \cdot 1 \cos \gamma+g_{x} \rho \frac{d x d y}{2}=\frac{d x d y}{2} \rho a_{x}$.

But $\cos \gamma=\frac{d y}{\sqrt{d x^{2}+d y^{2}}}$

Then taking the limit as the triangle shrinks to zero,

$P_{x} d y-P_{\gamma} d y=\operatorname{terms}$ of ordex $(d x)^{2}$

$\therefore P_{x}=P_{Y}$. But $Y$ is completely arbitrary.

Therefore the pressure is a scalar.

\section{LAWS OF FLUID MOTION}
(A) Conservation of momentum.
(B) Conservation of mass.
(C) Conservation of energy.

We make the assumption that the fluid flow is isentropic or that changes of state occur adiabatically. Hence we must consider the thermodynamic properties of the fluid that is, a connection between pressure, density, and energy. Therefore, we must add to the three conservation law a subsidiary equation, namely:

(D) Equation of State. 
The lawe mentioned above are sufficient to completely determine the properties of the fluid as a function of space and time.

\section{DERIVATION OF EQUATIONS OF LAWS OF FLUID MOTION}

There are two alternative methods of description both of which were originated by Euler. The two methods are known as the Eulerian representation and the Lagrangian representation.

\section{EUI.ERIAN DESCRIPTION}

In the Eulerian description the observer is fixed at a point in space, and $a s$ the fluid passes by he samples it, observing the density, velocity, and pressure as a function of time at that point. Therefore, from this description, by sampling at all points of the fluid, we know the properties of the fluid at all points in space at any given time. In this mode of description, the behavior of the fluid at our point of observation is influenced by its behavior at other points in the past. For example, the density at our point of observation at time $t$ will, in the case of a gas moving with uniform velocity, be the same as the density at a position in space $-v \Delta t$ from our observation point at the time $t-\Delta t$.

Thus, consider some property of the fluid $F=F(x, y, z, t)$.

If the gas has velocity components $u, v, w$ at the point $x, y, z$, at the time $t$, then at the time $t+\Delta t$

$$
F \rightarrow F(x+u \Delta t, y+v \Delta t, z+w \Delta t, t+\Delta t)
$$

and by a Taylor series expansion,

$F \rightarrow F(x, y, z, t)+u \Delta t\left(\frac{\partial F}{\partial x}\right)_{t}+v \Delta t\left(\frac{\partial F}{\partial y}\right)_{t}+w \Delta t\left(\frac{\partial F}{\partial z}\right)_{t}+\frac{\partial F}{\partial t} \Delta t=F(x, y, z, t)+\Delta F$. Then denoting $\frac{\Delta F}{\Delta t}$ by $\frac{D F}{D t}$

$$
\frac{D F}{D t}=\frac{\partial F}{\partial t}+\vec{u} \cdot \vec{F} \text {. }
$$

which is the Stoke s' notation for the total derivative in the Eulerian representation. 


\section{CONSERVATION LAWS IN EULERIAN REPRESENTATION}

\section{A. Conservation of Momentum}

Consider a rectangular parallelepiped of sides $\Delta x, \Delta y, \Delta z$, containing a fluid of density $\rho$, as shown in Fig. 3. Let the velocity components of the fluid be $u, v$, and $w$, and the pressure be $P$.

Then the amount of fluid in element $=\rho \Delta x \Delta y \Delta z$.

Consider the forces in $x$ direction: $F_{x}=m a_{x}=\rho \Delta x \Delta y \Delta z \frac{D u}{D t}$.

But

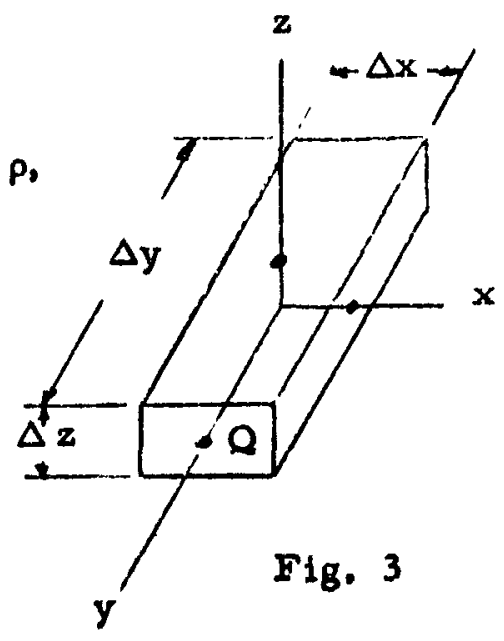
$F_{x}\left[P(x, y, z)-1 / 2 \Delta x \frac{\partial P}{\partial x}\right] \Delta y \Delta z-\left[P(x, y, z)+1 / 2 \Delta x \frac{\partial P}{\partial x}\right] \Delta y \Delta z$ $=-\frac{\partial p}{\partial x} \Delta x \Delta y \Delta z$

$$
\begin{aligned}
& \text { Similarly } F y=-\frac{\partial P}{\partial y} \Delta x \Delta y \Delta z \quad F_{z}=-\frac{\partial P}{\partial z} \Delta x \Delta y \Delta z \\
& \text { or in general } \frac{D L}{D t}=-\frac{1}{p} \vec{P} P .
\end{aligned}
$$

This is the equation for the conservation of momentum for a

fluid.

B. Conservation of Mass

Consider the parallelepiped above of volume $V$ at $t=t$.

The conservation of mass otates that

$$
\frac{D}{D t}(V \rho)=0 \text {, or } \frac{1}{\rho} \frac{D \rho}{D t}+\frac{1}{V} \frac{D V}{D t}=0 \text {. }
$$

At $t=t+\Delta t$ the coordinates of point $Q$ are

$$
\Delta x+\Delta x \frac{\partial u}{\partial x} \Delta t, \Delta y \frac{\partial v}{\partial y} \Delta t, \partial z \frac{\partial w}{\partial z} \Delta t \quad .
$$

Considering the volume of the parallelepiped at $t=t+\Delta t$ 


$$
\mathrm{V}(\mathrm{t}+\Delta \mathrm{t})=\Delta \mathrm{x} \Delta \mathrm{y} \Delta \mathrm{z}\left[\left(1+\frac{\partial u}{\partial \mathrm{x}} \Delta \mathrm{t}\right)\left(1+\frac{\partial \mathrm{v}}{\partial \mathrm{y}} \Delta \mathrm{t}\right)\left(1+\frac{\partial \mathrm{w}}{\partial \mathrm{t}} \Delta \mathrm{t}\right)\right]+
$$

second order cross products which $\rightarrow 0$ as the infinitesimals $\rightarrow 0$. Note that after the motion the parallelepiped is distorted, but its volume is still given by the projections above, namely $\vec{A} \times \vec{B} \cdot \vec{C}$.

$\therefore V(t+\Delta t)=V+\Delta V=\Delta x \Delta y \Delta z\left[1+\Delta t\left(\frac{\partial u}{\partial x}+\frac{\partial v}{\partial y}+\frac{\partial w}{\partial z}\right)\right]+$ second order terms or letting $\frac{\Delta V}{\Delta t}=\frac{D V}{D t}$

$$
\mathrm{V}+\left(\frac{\mathrm{D}}{\mathrm{D} t} \mathrm{~V}\right) \Delta \mathrm{t}=\Delta \mathrm{x} \Delta \mathrm{y} \Delta \mathrm{z}[\mathrm{l}+\vec{\nabla} \cdot \overrightarrow{\mathrm{u}} \Delta \mathrm{t}]
$$

But $\mathrm{V}=\Delta \mathrm{x} \Delta \mathrm{y} \Delta \mathrm{z}$ at $\mathrm{t}=\mathrm{t}$

or, $\frac{1}{\mathrm{~V}} \frac{\mathrm{DV}}{\mathrm{Dt}}=\vec{\nabla} \cdot \overrightarrow{\mathrm{u}}$

But $\rho V=$ mas $s=$ constant $\therefore \frac{1}{V} \frac{D V}{D t}=-\frac{1}{\rho} \frac{D \rho}{D t} \quad$ or

$$
\frac{D \rho}{D t}+\rho \vec{\nabla} \cdot \vec{u}=0 \text {. }
$$

which is the equation for the conservation of mass.

C. Conservation of Energy

From considerations similar to the above, one arrives at the following equation for the conservation of energy

$$
\frac{D}{D t}\left[\frac{1}{2} \vec{u} \cdot \vec{u}+E\right]=-\frac{1}{\rho}(\vec{\nabla} \cdot P \vec{u}) \text {. }
$$

where $E$ is the specific internal energy and thus $\frac{1}{2} \vec{u} \cdot \vec{u}+E$ corresponds to the total opecific energy, and $\vec{\nabla} \cdot P \vec{u}$ to an energy flow.

To summarize, we have four fundamental equations.

1. Conservation of Momentum.

2. Conservation of Mass.

3. Conservation of Energy.

4. Equation of State: $P=P(E, p)$ 


\title{
UNIVERSITY OF CALIFORNIA, RADIATION LABORATORY LIVERMORE SITE
}

LECTURES ON HYDRODYNAMICS AND SHOCK WAVES

\author{
Lectures by Robert Lelevier \\ Notes by Boris Ragent
}

March 12, 1954

Lecture 2: THE LAGRANGIAN REPRESENTATION. CONSERVATION LAWS IN THE LAGRANGIAN REPRESENTATION

\section{REVIEW}

In the last lecture, the question as to the correctness of excluding $p$ from the differentiations in the conservation equations arose. We will, therefore, give an alternate derivation which may help to clear up this point.

Consider a cell in a one-dimensional fluid as shown below. Let $\rho$ be the density, $u$ the velocity.

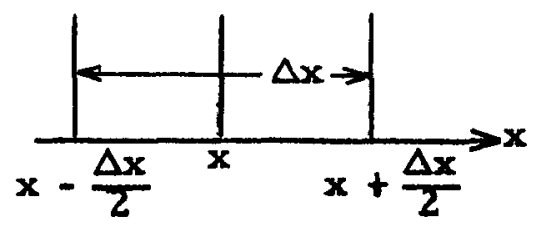

Then the momentum of the cell element is $p u \Delta x$

Time rate of change of momentum $=$ force

$$
\text { or } \begin{aligned}
\frac{\partial}{\partial t}(p u d x) & =[\underbrace{(\rho u) u-\frac{\Delta x}{2} \frac{\partial}{\partial x}\left(\rho u^{2}\right)}_{\text {Momentum flux into cell from left boundary }}] \\
& -\left[p u^{2}+\frac{\Delta x}{2} \frac{\partial}{\partial x}\left(\rho u^{2}\right)\right] \\
& +\left[P-\frac{\Delta x}{2} \frac{\partial p}{\partial x}\right]-\left[P+\frac{\Delta x}{2} \frac{\partial p}{\partial x}\right]
\end{aligned}
$$


Therefore we arrive at

$$
\frac{\partial}{\partial t}(p u)+\frac{\partial}{\partial x}\left(p u^{2}\right)+\frac{\partial p}{\partial x}=0 \text {. }
$$

which is the force equation in one dimension, and is the same equation as equation (1) in the last lecture, as we shall see.

From the last lecture we had

$$
\frac{\mathrm{D} \overrightarrow{\mathrm{u}}}{\mathrm{D} \mathrm{t}}+\frac{1}{\rho} \vec{\nabla} \mathrm{P}=0 \text {. }
$$

Expanding the above equation in one dimension (remembering that in Eulerian coordinates $\frac{D}{D t}=\frac{\partial}{\partial t}+\vec{u} \cdot \vec{\nabla}$

$$
p \frac{\partial u}{\partial t}+p u \frac{\partial u}{\partial x}+\frac{\partial p}{\partial x}=0
$$

Expanding equation (4) above we obtain

$$
p \frac{\partial u}{\partial t}+p u \frac{\partial u}{\partial x}+\frac{\partial p}{\partial x}+u\left[\frac{\partial p}{\partial t}+\frac{\partial(p u)}{\partial x}\right]=0 \text {. }
$$

But $\frac{\partial p}{\partial t}+\frac{\partial(p u)}{\partial x}=0$, since this is the equation of continuity.

$$
\therefore p \frac{\partial u}{\partial t}+\rho u \frac{\partial u}{\partial x}+\frac{\partial p}{\partial x}=0
$$

and the equations (1) and (4) are identical. Notice that the density is not involved in the above differentiations because of our assumption of conservation of mass.

\section{FURTHER REMARKS ON ENERGY CONSERVATION EQUATION}

We have assumed so far that we are dealing with continuous flow, i.e., that the functions in the differential equations are continuous and have a continuous first derivative. This implies that the flow is isentropic as one can see from the energy conservation equation.

For, consider the energy conservation equation

$$
\begin{aligned}
& \frac{D}{D t}\left[\frac{1}{2} \vec{u} \cdot \vec{u}+E\right]=-\frac{1}{\rho}(\vec{\nabla} \cdot P \vec{u}) \\
& \text { Since } \vec{u} \cdot\left[\frac{D \vec{u}}{D t}+\frac{1}{p} \vec{\nabla} P\right]=0 \text { (dotting } \vec{u} \text { into the force } \\
& \text { equation). } \\
& =\frac{D}{D t}\left[\frac{1}{2} \vec{u} \cdot \vec{u}\right]+\frac{1}{p}(\vec{u} \cdot \vec{\nabla} P) \\
& \text { we have }-\frac{1}{\rho}(\vec{u} \cdot \vec{\nabla} P)+\frac{D E}{D t}=-\frac{1}{\rho} P(\vec{\nabla} \cdot \vec{u})-\frac{1}{\rho}(\vec{u} \cdot \vec{\nabla} P)
\end{aligned}
$$


But from the conservation of mass $\vec{\nabla} \cdot \vec{u}=-\frac{1}{\rho} \frac{D p}{D t}$

therefore, conservation of energy equation becomes

$$
\begin{gathered}
\frac{D E}{D t}=\frac{P}{\rho^{2}} \frac{D \rho}{D t} \\
\text { or, } \frac{D E}{D t}=-P \frac{D V}{D t} \quad \text { since }-\frac{1}{\rho^{2}} \frac{D p}{D t}=\frac{D V}{D t} .
\end{gathered}
$$

But from the first law of thermodynamics

$$
\frac{D E}{D t}=-P \frac{D V}{D t}+T \frac{D S}{D t}
$$

or comparing the se equation $\mathrm{indicates}$ that $\frac{\mathrm{DS}}{\mathrm{Dt}}=0$, or that the entropy $s$ is a constant of the motion.

\section{EQUATION OF STATE AND SPEED OF SOUND}

In speaking of shocks and the velocity of propagation of shocks a knowledge of the speed of sound in the fluid is useful. For a perfect gas this speed is $c=\sqrt{\frac{\gamma P}{P}}$.

In calculating this velocity for an imperfect gas we must know the equation of state that connects three of the possible thermodynamic variables. The ve variables are:

$P=$ pressure

$\mathrm{T}=$ temperature

$V=\frac{1}{\rho}=$ specific volume

$S=$ entropy

e = specific energy.

Of the above five variables only two are independent. The equation of state specifies the relation between the se two independent variables (any two) and a third dependent variable. We usually choose $V$ and e as the independent variables, arriving at an equation for the dependent variable, $P$, of the form

$$
P=P(e, v)
$$


in which $P$ is usually a polynomial in $e$ and $V$.

\section{CALCULATION OF SPEED OF SOUND}

Consider a tube filled with gas. Make the customary assumption of isentropic flow. Now consider a disturbance being propagated to the right with a velocity $c$. Let $u$ be the fluid velocity to the left of the disturbance. Then the situation is indicated below:

\begin{tabular}{l|c}
\hline$P+\Delta P$ & \\
$\rho+\Delta \rho$ \\
$u>0$ \\
$u / c<<1$
\end{tabular}$\quad$\begin{tabular}{c} 
fluid at rest \\
\hline
\end{tabular}

Now consider an observer sitting on the boundary. Relative to him the fluid approaching him with velocity $c$ and the mass flowing toward him is $p c \mathrm{gms} / \mathrm{cm}^{2} / \mathrm{sec}$. The fluid flows away from him with velocity (c - u) and the mass flows away with a rate $(c-u)(p+\Delta p)$. By conservation of mass,

$$
p c=(p+\Delta p)(c-u) \equiv m
$$

also momentum is conserved. Therefore,

$$
\begin{aligned}
& m c-m(c-u)=P+\Delta P-P \quad \text { or } m u=\Delta P . \text { But } m=p c \\
& \therefore p c u=\Delta P
\end{aligned}
$$

If we now assume $u \Delta p<<c \Delta p$, we get from equations

(6) and (7)

$$
\begin{aligned}
\rho c & =p c+c \Delta p-u p-u \Delta p \\
\text { or } c \Delta p & =u p, \quad \text { but } u p=\frac{\Delta p}{c} \\
c^{2} & =\frac{\Delta p}{\Delta p} \\
\text { or } c & =\sqrt{\left(\frac{\partial p}{\partial p}\right)_{S}}
\end{aligned}
$$


If the equation of state does not provide $P$ as an explicit function of $\rho$ and $S$, then we must construct $\left(\frac{\partial p}{\partial p}\right)_{S}$ implicitly as follows:

Consider $P=P(e, V)$ :

Then $d P=\left(\frac{\partial P}{\partial e}\right)_{V} d e+\left(\frac{\partial P}{\partial V}\right)_{e} d V$

Consider $\mathrm{e}=\mathrm{e}(\mathrm{S}, \mathrm{V})$ :

Then de $=\left(\frac{\partial e}{\partial S}\right)_{V} d S+\left(\frac{\partial e}{\partial V}\right)_{S} d V$.

$\therefore d P=\left(\frac{\partial P}{\partial e}\right)_{V}\left(\frac{\partial e}{\partial S}\right)_{V} d S+\left(\frac{\partial P}{\partial e}\right)_{V}\left(\frac{\partial e}{\partial V}\right)_{S} d V+\left(\frac{\partial P}{\partial V}\right)_{e} d V$.

From the first law of thermodynamics

$$
\begin{aligned}
& \text { de }=\mathrm{TdS}-\mathrm{PdV} \\
& \text { or } \quad T=\left(\frac{\partial e}{\partial S}\right)_{V} \text { and } P=-\left(\frac{\partial e}{\partial V}\right)_{S} \\
& \therefore d P=\left(\frac{\partial P}{\partial e}\right)_{V} T d S+d V \quad\left[\left(\frac{\partial P}{\partial V}\right)_{e}-P\left(\frac{\partial P}{\partial e}\right)_{V}\right] \\
& c^{2}=\left(\frac{\partial p}{\partial p}\right)_{S}=-V^{2}\left(\frac{\partial P}{\partial V}\right)_{S} \text {, since } \rho=\frac{1}{V} \text { and } d p=-\frac{1}{V^{2}} d V \\
& \text { hence } c^{2}=-V^{2}\left(\frac{\partial P}{\partial V}\right)_{e}+P V^{2}\left(\frac{\partial P}{\partial e}\right)_{V} \text {. }
\end{aligned}
$$

\section{Example - Perfect Gas}

For a perfect gas

$$
\begin{aligned}
P V & =R T \\
& =\left(c_{P}-c_{V}\right) T \\
& =(\gamma-1) c_{V} T=(\gamma-1) \int_{0}^{T} c_{V} d T \\
& =(\gamma-1) e \\
P & =\frac{(\gamma-1) e}{V} \\
\frac{\partial p^{\prime}}{\partial V} & =-\frac{(x-1) e}{V^{2}}, \frac{\partial P}{\partial e}=\frac{\gamma-1}{V} \\
C^{2} & =(\gamma-1) e+P V(\gamma-1)
\end{aligned}
$$




$$
\begin{aligned}
& =(\gamma-1)(e+P V) \\
& =(\gamma-1)\left(\frac{1}{\gamma-1}+1\right) P V \\
C^{2} & =\gamma P V \quad \text { or } \quad c=\sqrt{\frac{\gamma P}{P}}
\end{aligned}
$$

\section{THE LAGRANGIAN REPRESENTATION}

This method of description differs from the previous Euler method in that instead of remaining fixed in position and confining our attention to the flow through that position, we will here follow the timespace history of every particle in the fluid. Thus, we label each particle and follow it in time, observing what happens to the pressure, entropy, energy, density, etc., at the point at which our particle happens to be at that time. Therefore, our Lagrangian coordinates will be labels or indices attached to points. This labeling can be arbitrary; however, a convenient choice is to take as the Lagrange coordinates the initial position of the particles.

Example - Two-Dimensional Gas

Consider a particle in a gas which has the coordinates

$$
(a, b) \text { at } t=0 \text {. At any later time the coordinates of the }
$$

particle will be

$$
\left.\begin{array}{l}
x=x(a, b, t) \\
y=y(a, b, t)
\end{array}\right\}
$$

Given the initial coordinate $s(a, b)$ of a particle, the tranaformation (11) give it position $(x, y)$ at a later time, $t$.

The components of the velocity of a particle are,

$$
\left.\begin{array}{ll}
u=\left(\frac{\partial x}{\partial t}\right)_{a, b \text { constant }}=u(a, b, t) \\
v=\left(\frac{\partial y}{\partial t}\right)_{a, b \text { constant }}=v(a, b, t)
\end{array}\right\} \quad \begin{aligned}
& \text { time rate of change } \\
& \text { of the position } \\
& \text { of the particle }
\end{aligned}
$$

We can, in principle, solve the equation (II) for $a$ and $b$ as functions of $x, y$ and $t, i, e$. 


$$
\begin{aligned}
& a=a(x, y, t) \\
& b=b(x, y, t) .
\end{aligned}
$$

The Jacobian of the transformation is

$$
J=\left|\begin{array}{ll}
\frac{\partial x}{\partial a} & \frac{\partial y}{\partial a} \\
\frac{\partial x}{\partial b} & \frac{\partial y}{\partial b}
\end{array}\right|
$$

By the rules of implicit differentiation we have,

$$
\left.\begin{array}{lll}
\frac{\partial a}{\partial x}=\frac{1}{J} \frac{\partial y}{\partial b} & ; & \frac{\partial b}{\partial x}=-\frac{1}{J} \frac{\partial y}{\partial a} \\
\frac{\partial a}{\partial b}=-\frac{1}{J} \frac{\partial x}{\partial b} ; & \frac{\partial b}{\partial y}=\frac{1}{J} \frac{\partial x}{\partial a}
\end{array}\right\} .
$$

\section{THE CONSERVATION EQUATIONS}

Proceeding as in the last lecture, we derive the equation of momentum conservation (in the $x$-direction),

$$
\frac{\partial^{2} x}{\partial t^{2}}=-\frac{1}{\rho} \frac{\partial p}{\partial x}
$$

But, remembering that $P=P(a, b, t)$

$$
x=x(a, b, t) \text {. }
$$

we must transform to our variables $a, b$, and $t$.

(Remembering that the point $s a, b$ are independent of the time)

$$
\begin{aligned}
\text { Now, }\left[\frac{\partial f(a, b, t)}{\partial x}\right]_{t}=\text { const } & =\frac{\partial f}{\partial a} \frac{\partial a}{\partial x}+\frac{\partial_{f}}{\partial b} \frac{\partial b}{\partial x} \\
\therefore \quad \frac{\partial^{2} x}{\partial t^{2}} & =\frac{1}{\rho}\left(\frac{\partial p}{\partial a} \frac{\partial a}{\partial x}+\frac{\partial p}{\partial b} \frac{\partial b}{\partial x}\right) \\
& =-\frac{1}{p} \frac{1}{J}\left(\frac{\partial p}{\partial a} \frac{\partial y}{\partial b}-\frac{\partial p}{\partial b} \frac{\partial y}{\partial a}\right) .
\end{aligned}
$$

Let the notation $[P, y]$ stand for $\left(\frac{\partial p}{\partial a} \frac{\partial y}{\partial b}-\frac{\partial p}{\partial b} \frac{\partial y}{\partial a}\right)$

Therefore we have $\frac{\partial u}{\partial t}+\frac{1}{\rho J}[P, y]=0$, 
where $u=\frac{\partial x}{\partial t}$.

In the other dimensions, we have

$$
\frac{\partial v}{\partial t}+\frac{1}{p J}[x, P]=0 \text {. }
$$

(Note the inversion in the brackets.)

These are the force equations for a two-dimensional gas.

\section{CONSERVATION OF MASS}

Consider $\frac{\partial u}{\partial x}+\frac{\partial v}{\partial y}$, the divergence.

$$
\begin{array}{r}
\frac{\partial u}{\partial x}+\frac{\partial v}{\partial y}=\frac{1}{J}\left(\frac{\partial u}{\partial a} \frac{\partial y}{\partial b}-\frac{\partial u}{\partial b} \frac{\partial y}{\partial a}-\frac{\partial v}{\partial a} \frac{\partial x}{\partial b}+\frac{\partial v}{\partial b} \frac{\partial x}{\partial a}\right) \\
\text { Now } \frac{\partial}{\partial t}\left(\frac{\partial x}{\partial a} \frac{\partial y}{\partial b}\right)=\frac{\partial u}{\partial a} \frac{\partial y}{\partial b}+\frac{\partial x}{\partial a} \frac{\partial v}{\partial b} \text { by inverting }
\end{array}
$$

the order of differentiation, since we have continuity.

therefore we have $\frac{\partial v}{\partial y}+\frac{\partial u}{\partial x}=\frac{1}{J} \frac{\partial J}{\partial t}$

The fundamental connection between the Euler and Lagrange representation is

$$
\bar{\nabla} \cdot \overrightarrow{\mathrm{u}}=\frac{1}{\mathrm{~J}} \frac{\partial \mathrm{J}}{\partial \mathrm{t}} \text {. }
$$

The conservation of mass equation reade

$$
\frac{\partial \rho}{\partial t}+\rho \vec{\nabla} \cdot \vec{u}=0 \text {. }
$$

Note that the $\vec{u} \cdot \vec{\nabla} \mathbf{p}$ term present in the Euler dencription is absent here.

We can also write the above

$$
\frac{\partial p}{\partial t}+\frac{p}{J} \frac{\partial J}{\partial t}=0
$$

or dividing by $\rho$ and integrating we get,

$$
\ln \rho \mathrm{J}=\text { const or } \rho \mathrm{J}=\text { constant }
$$


But at $t=0, J=1$ (since $x=a, y=b$ ), hence, the constant of integration is

$$
\begin{aligned}
p(a, b, 0) & \equiv p_{0}(a, b) . \\
\therefore p J & =p_{0}(a, b) .
\end{aligned}
$$

This is the conservation of mass equation in Lagrange coordinates. Using this we can simplify the force equations to read

$$
\left.\begin{array}{c}
\frac{\partial u}{\partial t}+\frac{1}{p_{0}}[p, y]=0 \\
\text { and } \frac{\partial v}{\partial t}+\frac{1}{p_{o}}[x, P]=0
\end{array}\right\} \text {. }
$$

Note that if the fluid is incompressible, $p=$ constant and $\mathrm{J}=1$ for all time.

In like manner, we could derive the energy conservation equation which is stated below.

$$
\frac{\partial E}{\partial t}+P \frac{\partial V}{\partial t}=0 \text { Conservation of Energy }
$$

\section{SUMMARY}

In Lagrangian coordinates we have the following equations:

$$
\begin{aligned}
& \frac{\partial u}{\partial t}+\frac{1}{\rho_{o}}[P, y]=0 \\
& \left.\frac{\partial v}{\partial t}+\frac{1}{\rho_{0}}[\mathbf{x}, \mathbf{P}]=0\right\} \text { Conservation of Momentum } \\
& \text { where } \quad \frac{\partial x}{\partial t}=u \quad \frac{\partial y}{\partial t}=v \\
& \rho J=p_{0} \quad \text { Conservation of Mass } \\
& \frac{\partial E}{\partial t}+\rho \frac{\partial v}{\partial t}=0 \quad \text { Conservation of Energy } \\
& P=P(e, v) \quad \text { The equation of state. }
\end{aligned}
$$




\title{
UNIVERSITY OF CALIFORNIA, RADIATION LABORATORY LIVERMORE SITE
}

\section{LECTURES ON HYDRODYNAMICS AND SHOCK WAVES}

\author{
Lectures by Robert Lelevier - Notes by Boris Ragent
}

March 19, 1954

\section{Lecture 3: SHOCK WAVES, RANKINE - HUGONIOT CONDITIONS, APPROXIMATIONS TO ADIABATIC FLOW}

Before discussing shock waves we should note, that with reference to the two previous lectures, for multi dimensional problems it is sometimes convenient to use a mixed or pseudo representation. For example, in the case of a two-dimensional gas, we might use a Lagrange representation in the $\mathrm{x}$-direction and an Euler representation in the $\mathrm{y}$-di=rection.

\section{DEVELOPMENT OF SHOCKS}

A qualitative description of the way in which shocks develop may be made as follows:

Consider a cylinder containing a fluid and piston arrangement as shown in Fig. 4.

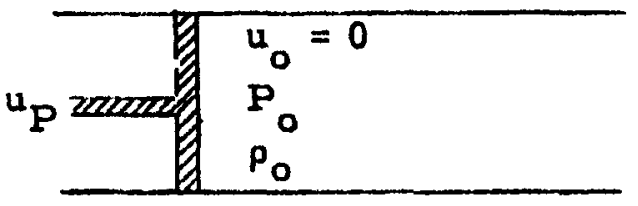

Suppose the piston is accelerated to the right as indicated schematically in Fig. 4 , the initial conditions of the fluid being $u_{0}=0, P=P_{0}$ and $p=P_{0} \cdot$ As the piston begins to move, a small volume of fluid in front of the plunger is comprossed, starting a disturbance moving down the cylinder to the right with a velocity characteristic of the velocity

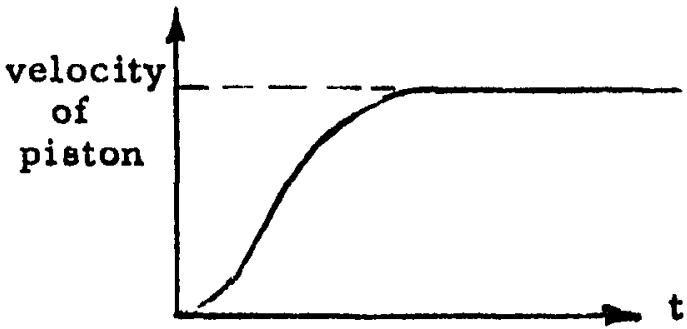

Fig. 4 
of propagation in the undisturbed fluid. The disturbance in moving down the cylinder will heat the fluid, raising its temperature. Now if we consider the motion of the piston at a short time after the piston has begun its motion, we can see that the mass of fluid being compressed by the piston now, and its associated disturbance are moving in a medium whose temperature is higher than it was initially. The velocity of propagation (velocity of sound) being an increasing function of temperature we can see that this second disturbance will tend to overtake the first. Further motion of the piston then sends out disturbances moving with greater velocities than the previous disturbances, and eventually a condition is reached in which the earlier disturbances are overtaken. At this point we apparently have the fluid sustaining two different sound speeds. This is impossible if the flow were to remain continuous $\left(c^{2}=(\partial P / \partial \rho)_{S}\right)$. Hence a discontinuity is established which propagates down the tube. This discontinuity is referred to as a "shock".

Real shocks, of course, have finite thickness, (commonly of the order of fractions of a millimeter). However, one can idealize the treatment of shock fronts by means of mathematical discontinuities.

Experimentally, shock waves have been studied in several places. In Berkeley, for example, there is an apparatus used in their wind tunnel capable of creating shocks which are approximately one-quarter inch in thickness. At Harvard, Horning has studied shock wave thickvesses using the apparatus shown in Fig. 5.

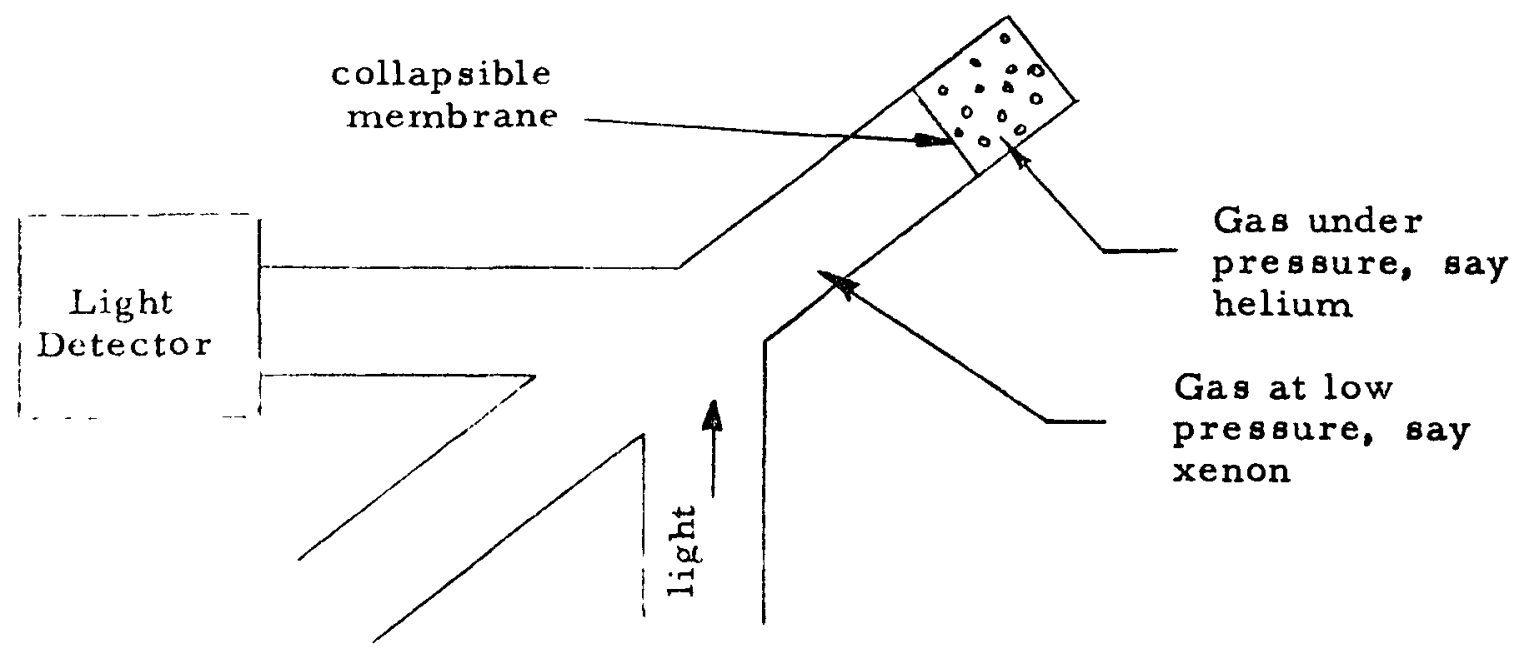

Fig. 5 
By measuring the reflection of light from the shock front due to changes of the index of refraction of the gas, he has measured reflection coefficients of $\sim 10^{-6}$ corresponding to shock front thicknesses of approximately one millimeter.

\section{THE RANKINE - HUGONIOT CONSERVATION EQUATIONS}

Consider a one-dimensional gas containing a discontinuity moving to the right with velocity $u$. Let the parameters

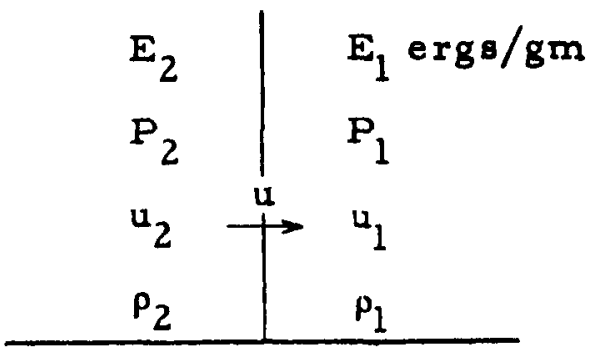

of the gas to the right of the discontinuity be characterized by the subscript 1 , and those to the left be characterized by the subscript 2 .

Suppose we take the position of observers on the discontinuity front. By conservation of matter, matte $r$ coming toward observer $/ \mathrm{cm}^{2} / \mathrm{sec}$ $=$ matter leaving observer $/ \mathrm{cm}^{2} / \mathrm{sec}$

$$
\text { or } m=\left(u_{1}-u\right) p_{1}=\left(u_{2}-u\right) p_{2}
$$

This is the equation for the conservation of mass.

By the conservation of momentum,

$$
\begin{gathered}
\text { nomentum coming toward observer - momentum leaving } \\
\text { observer }
\end{gathered}
$$

$=$ difference in pressure

$$
\therefore m\left(u_{1}-u\right)-m\left(u_{2}-u\right)=P_{2}-P_{1} \text {, }
$$


which is the equation for conservation of momentum.

Similarly, by conservation of energy, total energy on right - total energy on left = total work done

or

$$
m\left[\frac{1}{2} u_{1}^{2}+E_{1}\right]-m\left[\frac{1}{2} u_{2}^{2}+E_{2}\right]=P_{2} u_{2}-P_{1} u_{1}
$$

Notice that the structure of the equation here is analogous to that of the continuous case.

$$
\frac{D}{D t}\left[\frac{1}{2} u^{2}+E\right]=-\frac{1}{\rho} \vec{\nabla} \cdot(P \vec{u})
$$

Equations (23), (24), and (25) above constitute the Rankine Hugoniot equations in one dimension.

The form of the equations above is not particularly convenient. With a little algebra we arrive at the forms

$$
\begin{aligned}
& u_{2}=u_{1}+\left[\frac{\left(P_{2}-P_{1}\right)\left(\rho_{2}-p_{1}\right)}{\rho_{1} \rho_{2}}\right]^{1 / 2} \\
& u=\frac{u_{2} p_{2}-u_{1} p_{1}}{p_{2}-p_{1}} \\
& E_{2}-E_{1}=\frac{1}{2}\left(P_{1}+P_{2}\right)\left[\frac{1}{p_{1}}-\frac{1}{p_{2}}\right]
\end{aligned}
$$

Equation (23a) gives the fluid velocity behind the shock front. Equation (24a) give $s$ the velocity of the shock wave, and equation (25a) is of fundamental importance, expressing the difference in specific energy across the shock front. The form of equation $(25 \mathrm{a})$ is analogous to the adiabatic case $\Delta E=-P \Delta\left(\frac{1}{\rho}\right)$.

The Hugoniot Curve

Suppose that we have for the equation of state of a fluid $P=P(E, p)$. Now, if the fluid is shocked, we can expect the behavior of the fluid to follow the behavior illustrated in the curve in Fig. 6

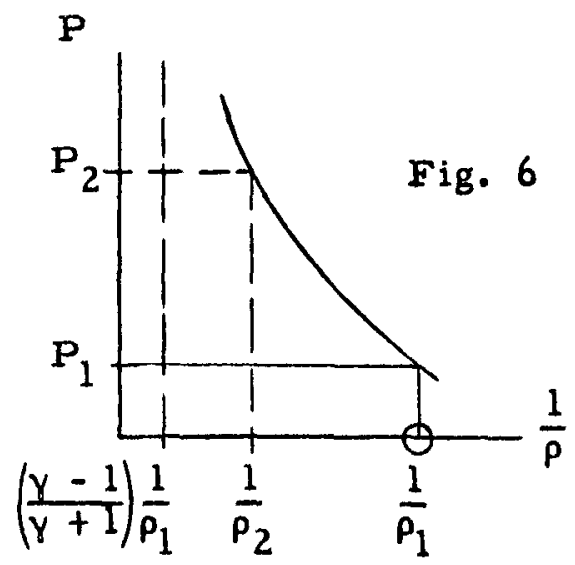


The curve is a locus of point s called the Hugoniot curve, obtained by eliminating $E$ from the equation (25a) and the equation of state. This locus of points describes the possible states in which a fluid may end upon being shocked. For a perfect fluid the line at $\left(\frac{\gamma-1}{\gamma+I}\right) \frac{1}{p_{1}}$ is the Hugoniot for a shock starting from the state $P=0 \quad p=p_{1}$ (indicated by the circle in Fig. 6).

Example - Perfect Gas

Consider a $\gamma$-law gas for which

$$
E=\frac{P}{(\gamma-1) \rho}
$$

Let $\eta=\frac{\rho_{2}}{\rho_{1}}, y=\frac{P_{2}}{P_{1}} ; P_{2}, P_{2}$ are final conditions $; P_{1}, p_{1}$ are initial conditions. Using the se substitutions in equations (23a), (24a), and (25a) above, we obtain,

$$
\eta=\frac{y(\gamma+1)+(\gamma-1)}{y(\gamma-1)+(\gamma+1)}
$$

and $u_{2}=u_{1}+\left[\frac{2 P_{1}}{\rho} \frac{(y-1)^{2}}{y(y+1)+(\gamma-1)}\right]^{1 / 2}$

For strong shocks, $y>>1$

and $u_{2} \longrightarrow u_{1}+\sqrt{\frac{2}{\gamma+1} \frac{P_{2}}{\rho_{1}}}$

It is interesting to note the limiting value of $\frac{1}{p_{2}}$, that is $\left(\frac{\gamma-1}{\gamma+1}\right) \frac{1}{\rho_{1}}$. This is the maximum possible compression of a fluid over one shock.

Suppose we have a small pressure differential, such that $y=1+\epsilon$ where $E$ is small

$$
\begin{aligned}
\therefore \eta \cong 1+\frac{E}{\gamma} \text { since } \eta & =\frac{y(\gamma+1)+(\gamma-1)}{y(\gamma-1)+(\gamma+1)} \\
& \approx \frac{2 y+(1+\gamma) E}{2 \gamma+(\gamma-1)}
\end{aligned}
$$




$$
\begin{aligned}
& \approx\left[1+\frac{(\gamma+1)}{2 \gamma} E\right]\left[1-\frac{(\gamma-1)}{2 \gamma} E\right] \\
& \approx 1+\frac{\epsilon}{\gamma} .
\end{aligned}
$$

For an adiabatic compression for a perfect gas

$$
\eta=y^{\frac{1}{\gamma}}=\left(1+\epsilon^{\frac{1}{\gamma}} \cong 1+\frac{1}{\gamma} \epsilon+\ldots \cdot\right.
$$

Therefore, in the limit of weak shocks we have adiabatic compression. The validity of this approximation is illustrated in Table I below.

Table I. COMPARISON OF COMPRESSIONS FOR ADIABATIC AND SHOCK CONDITIONS

\begin{tabular}{|ccc|}
\hline For a $\gamma=\frac{5}{3}$ gas, & & \\
$y$ & $\eta$ adiabatic & $\eta$ shock \\
\hline 1 & 1 & 1 \\
1.5 & 1.275 & 1.270 \\
2 & 1.516 & 1.500 \\
5 & 2.625 & 2.333 \\
10 & 3.950 & 2.930 \\
\hline
\end{tabular}

Therefore, the adiabatic approximation to compression is fairly gcod up to overpressures of a factor of 2 . 


\section{UNIVERSITY OF CALIFORNIA, RADIATION LABORATORY LIVERMORE SITE}

LECTURES ON HYDRODYNAMICS AND SHOCK WAVES

Lectures by Robert Lelevier - Notes by Boris Ragent

April 2 and 23, 1954

Lecture 4: REFLECTIONS OF SHOCKS FOR NORMAL INCIDENCE. METHOD OF CHARACTERISTICS FOR ONE-DIMENSIONAL

FLOW

\section{INT RODUCTION}

The following discussion gives a brief intuitive description of some of the concepts involved in the propagation of a shock.

Consider the behavior of a shock tube as shown in one dimension, in Fig. 7, when the membrane is broken.

Pressure

At the instant that the membrane is broken the high pressure gas starts expanding into the low pressure gas with an interface, behaving like a piston, and producing a shock in the low pressure gas. The pressure interface thus produced moves to the right with a velocity characteristic of the velocity of sound in the unshocked gas. However, the shock produced travels at a velocity characteristic of the shock velocity in the unshocked gas: a velocity which is greater than the velocity of the interface. Therefore, the pressure profile extends at any given instant farther into the gas than the interface position. Simultaneously the pressure behind the interface falls adiabatically (since there is no shock wave progressing to the left) starting a rarefaction fan moving back into the left hand medium with a velocity 


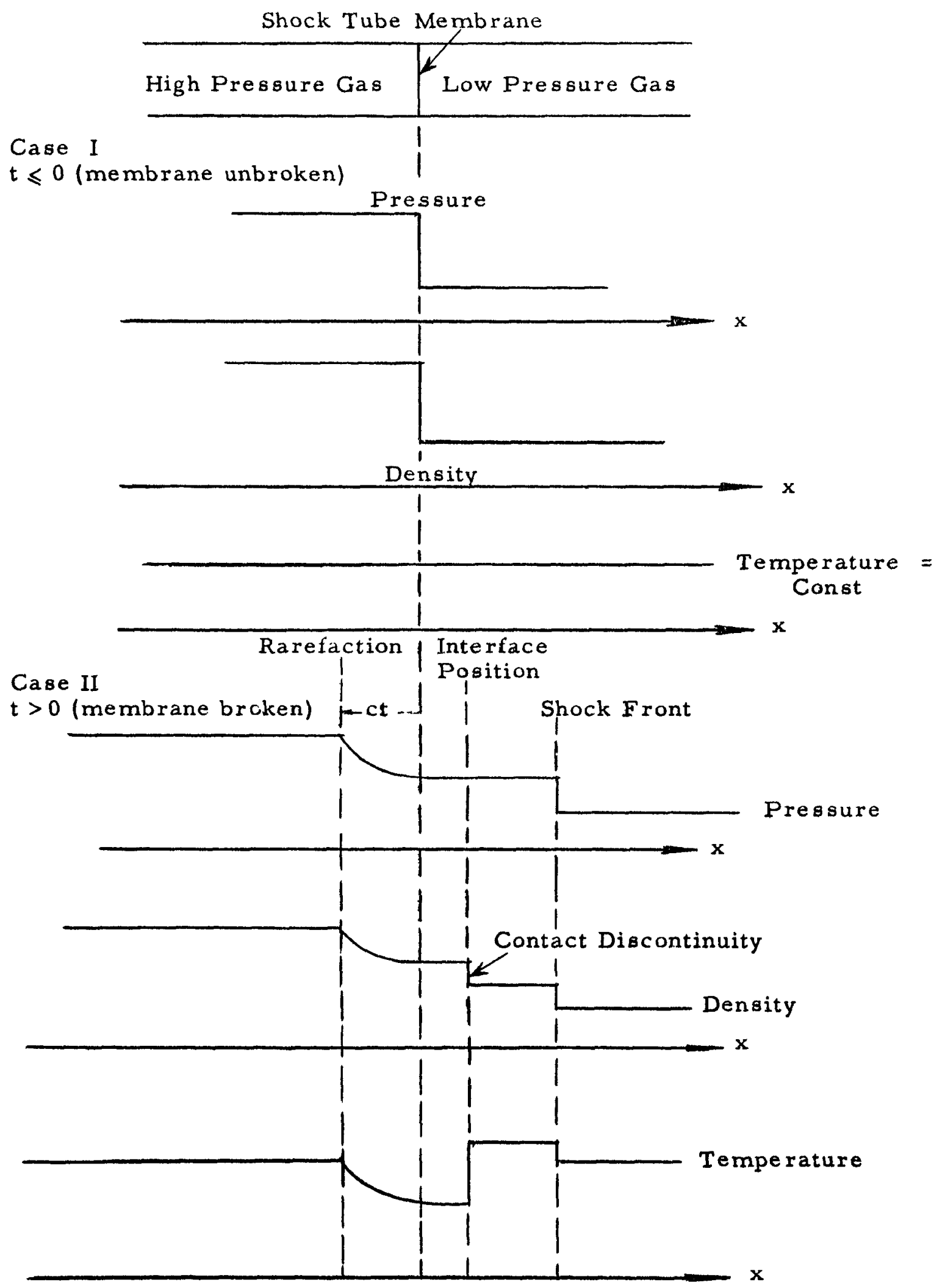

Fig. 7 
characteristic of the velocity of sound in the high pressure gas. The pressure profile can be calculated using the Riemann method of characteristics to be discussed later.

$\underline{\text { Density }}$

The density profile after the membrane is broken will appear to have a discontinuity at the position that the interface has assumed. This can be visualized by considering that the density ahead of the interface must be greater than that of the unshocked material, but still must be less than that of the compressing material. Behind this interface the density must, of course, rise to the initial density of the high pressure gas.

Temperature

Here again the temperature of the shocked gas must be higher than the temperature of either the high or low pressure gases which were originally in equilibrium. The temperature ahead of the interface is therefore higher than the equilibrium temperature, whereas the temperature behind the interface is lower, having been cooled by the expansion. Again here the temperature at the left rises to the equilibrium temperature at the point which the rarefaction fan has reached.

The profiles of pressure, density, and temperature are illustrated below, (Fig. 7). Note that in an actual case we will have some diffusion, but the diffusion times are, in general, fairly long compared with the times under consideration, and the fronts of the profile maintain their character fairly well.

In the discussion above we have assumed that the shock velocity was greater than the velocity of sound (of the interface) in the gas. This is strictly true, in the case of a $\gamma$-law gas only for $\gamma<2$.

For, from the last lectures, equation (28), the shock speed is $u=\sqrt{\frac{2}{\gamma+1} \frac{P_{0}}{P_{0}}}$, whereas for strong shocks from equations $(10)$ and $(28)$ the sound speed is $c=\sqrt{\frac{\gamma P_{0}}{P}}=\sqrt{\frac{\gamma P_{0}(\gamma-1)}{P_{0}(\gamma+1)}}$, 


$$
\begin{aligned}
& \therefore \frac{u}{c}=\left\{\frac{2}{\gamma+1} \frac{\gamma+1}{\gamma(\gamma-1)}\right\}^{1 / 2}=\left[\frac{2}{\gamma(\gamma-1)}\right]^{1 / 2} \\
& \therefore \text { for } \gamma=2 \quad u=c \\
& y<2 \quad u>c \quad \text { or supersonic flow } \\
& \gamma>2 \quad u<c \quad \text { or subsonic flow. }
\end{aligned}
$$

REFLECTIONS OF SHOCKS FOR NORMAL INCIDENCE

The problem of shock reflections in the case of normal incidence can be solved analytically. The case of oblique reflections, which can involve the formation of Mach stems, is treated in the reference article "Interaction of Shock Waves" by Bleakney and Taub, given in Lecture 1.

Consider two media, $A$ and $B$, with a shock in medium $A$ traveling toward the interface as shown in Fig. 8. We assume $P_{A_{0}}<\rho_{B_{0}}$ where the se are the initial unshocked densities

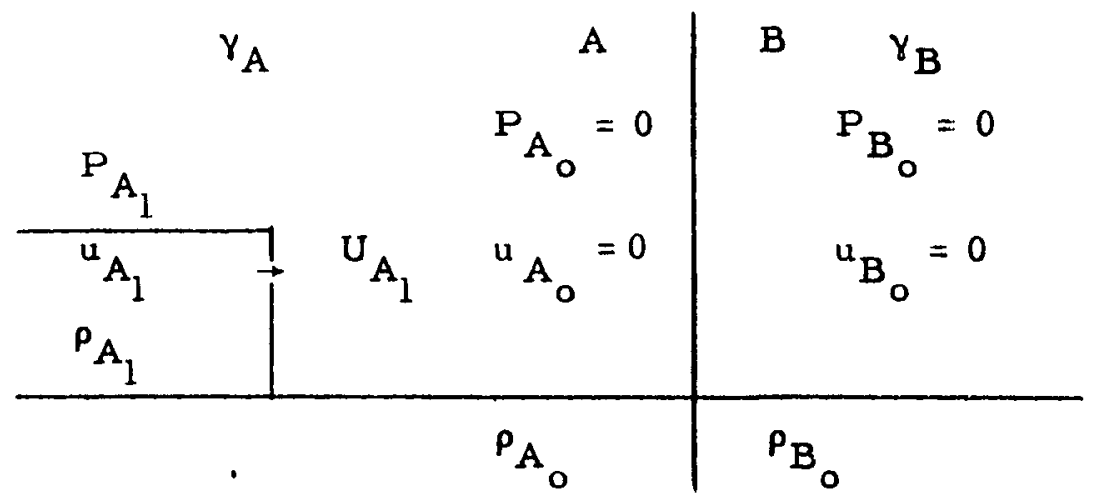

Fig. 8

From the strong shock conditions we have

$$
\begin{aligned}
& \rho_{A_{1}}=\frac{\gamma_{A}+1}{\gamma_{A}-1} \quad \rho_{A_{0}} \\
& u_{A_{1}}^{2}=\frac{2}{\gamma_{A}+1} \frac{P_{A_{1}}}{P_{A_{0}}}
\end{aligned}
$$


When the shock impinges on the interface, reflection occurs giving rise to a pressure distribution as shown in Fig. 9.

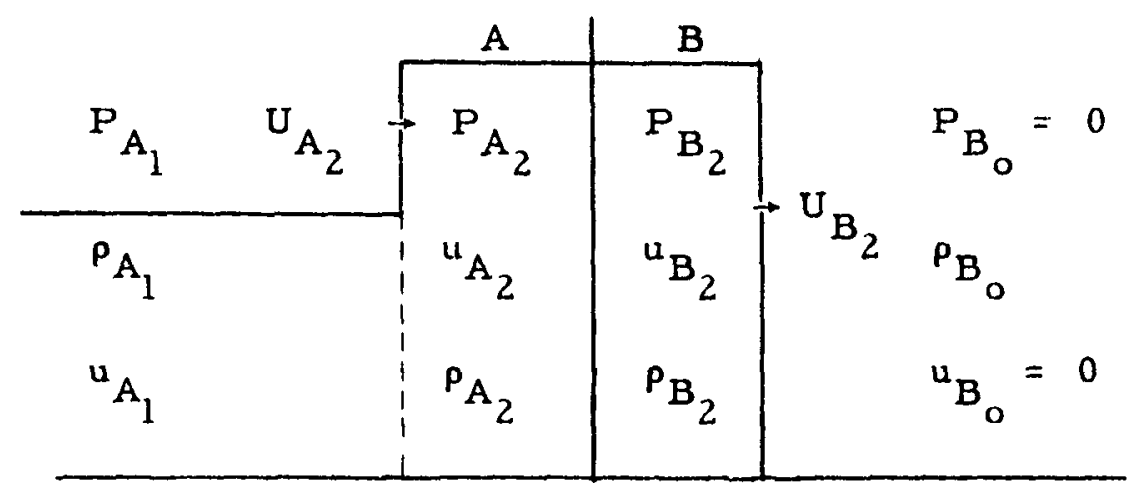

Fig. 9

Across the interface we have $\mathrm{u}_{\mathrm{A}_{2}}=\mathrm{u}_{\mathrm{B}_{2}}$ and $\mathrm{P}_{\mathrm{A}_{2}}=\mathrm{P}_{\mathrm{B}_{2}} \equiv \mathrm{P}$.

The problem is to determine $P$, the pressure on reflection, given $P_{A_{1}}, \rho_{A_{0}}$ and $\rho_{B_{0}}$. The procedure is to determine $u_{A_{2}}$, using the Hugoniot conditions, across the reflected shock and, similarly, $u_{B_{2}}$ across the transmitted shock. Equating ${ }_{A_{2}}$ and $u_{B_{2}}$ yields a transcendental equation for $P$ in terms of the ratio $p_{B_{0}} / p_{A_{0}}$. We have

$$
\begin{aligned}
& u_{A_{2}}=u_{A_{1}}-\sqrt{P-P_{A_{1}}} \sqrt{\frac{P_{A_{2}}-P_{A_{1}}}{P_{A_{2}} P_{A_{1}}}} \\
& \frac{P_{A_{2}}}{P_{A_{1}}}=\frac{y\left(\gamma_{A}+1\right)+\left(\gamma_{A}-1\right)}{y\left(\gamma_{A}-1\right)+\left(\gamma_{A}+1\right)}
\end{aligned}
$$

where $y=\frac{P}{P_{A_{1}}}$,

and $u_{B_{2}}=\sqrt{\frac{2}{Y_{B}+1} \frac{P}{\rho_{B_{0}}}}$. 
If we let $x=\rho_{B_{0}} / \rho_{A}$ then the procedure outlined gives

$$
\sqrt{y} \equiv \sqrt{\frac{\gamma_{B}+1}{\gamma_{A}+1}} \sqrt{x}\left\{1-(y-1) \sqrt{\frac{\gamma_{A}-1}{y\left(\gamma_{A}+1\right)+\gamma_{A}-1}}\right\}
$$

In the case of reflection from a rigid wall, $x=\infty$

hence

$$
\begin{aligned}
& 1-(y-1) \sqrt{\frac{\gamma_{A}-1}{y\left(\gamma_{A}+1\right)+\gamma_{A}-1}}=0 \\
& y=\frac{3 \gamma_{A}-1}{\gamma_{A}-1} .
\end{aligned}
$$

If $x=1$ and $\gamma_{B}=\gamma_{A^{\prime}}, y=1$.

If $x=1$ and $\gamma_{B}>\gamma_{A}$ (Material $B$ is "stiffer" than material $A$ )

then we have a solution $y>1$.

If $x=1$ and $\gamma_{B}<\gamma_{A}$, the equation has a nonphysical root $0<y<1$. In this case a rarefaction occurs in "A" preceeded by a shock in "B".

\section{METHOD OF CHARACTERISTICS FOR ONE-DIMENSIONAL FLOW}

The method of characteristics using the Riemann invariants is very useful for calculations in the one-dimensional case, that is, one Cartesian space coordinate. In more than one dimension, or in non-Cartesian coordinates, the method is not particularly convenient.

The method seeks to find a curve in the $(t, x)$ plane, a characteristic, such that along this curve some function, the characteristic function, is a constant. For example, in a one-dimensional isentropic flow we have the conservation equations,

$$
\frac{\partial u}{\partial t}+u \frac{\partial u}{\partial x}+\frac{c^{2}}{\rho} \frac{\partial \rho}{\partial x}=0
$$

the momentum conservation equation, and

$$
\frac{\partial \rho}{\partial t}+u \frac{\partial \rho}{\partial x}+\rho \frac{\partial u}{\partial x}=0
$$

the mass conservation equation. 
Suppose that $\sigma$ is some curve in the $(t, x)$ plane. We now attempt to find a function $F$ such that

$$
\frac{d}{d \sigma} F(p, u)=\frac{\partial F}{\partial \rho} \frac{d \rho}{d \sigma}+\frac{\partial F}{\partial u} \frac{d u}{d \sigma}=0
$$

Then $F=$ constant along $\sigma$ and if we can determine $F$ at $t=0$ from the initial condition s we have a plot of a function characteristic of the flow.

Development of Method

Consider a continuous (but perhaps non-isentropic) flow in one dimension, the $x$-dimension. The equations of motion for the flow are

$$
\begin{aligned}
& \frac{\partial u}{\partial t}+u \frac{\partial u}{\partial x}+\frac{1}{p} \frac{\partial P}{\partial x}=0, \text { or since } c^{2}=\frac{\partial P}{\partial p}, \\
& \frac{1}{p} \frac{\partial P}{\partial x}=\frac{c^{2}}{p} \frac{\partial p}{\partial x},
\end{aligned}
$$

and

$$
\frac{\partial u}{\partial t}+u \frac{\partial u}{\partial x}+c^{2} \frac{\partial p}{\partial x}=0 \text {, }
$$

the mass conservation equation,

and

$$
\frac{\partial \rho}{\partial t}+u \frac{\partial \rho}{\partial x}+\rho \frac{\partial u}{\partial x}=0 \text {, }
$$

the momentum conservation equation.

$$
\text { Multiplying equation (34) by } \frac{c}{p} \text { and adding to equation (33) we }
$$

obtain

$$
\frac{c}{p} \frac{\partial p}{\partial t}+u \frac{c}{p} \frac{\partial p}{\partial x}+c \frac{\partial u}{\partial x}+\frac{\partial u}{\partial t}+u \frac{\partial u}{\partial x}+c \frac{c}{p} \frac{\partial p}{\partial x}=0 .
$$

Let $\mathrm{u} s$ introduce the quantity $\omega=\omega(x, t)$ defined as

$$
\omega(x, t)=\int^{\rho} c \frac{d \rho}{\rho} .
$$

Then

$$
\frac{\partial \omega}{\partial x}=\frac{c}{\rho} \frac{\partial \rho}{\partial x}
$$

and

$$
\frac{\partial \omega}{\partial t}=\frac{c}{p} \frac{\partial p}{\partial t}
$$

Therefore equation (35) becomes,

$$
\frac{\partial \omega}{\partial t}+\frac{\partial u}{\partial t}+(u+c)\left(\frac{\partial \omega}{\partial x}+\frac{\partial u}{\partial x}\right)=0 \text {. }
$$


Now if we let $r=\frac{\omega+u}{2}$ equation (36) becomes

$$
\frac{\partial \mathbf{r}}{\partial t}+(c+u) \frac{\partial r}{\partial x}=0
$$

Equation (37) states that lines of constant $r$ are propagated with a velocity $c+u$ in the $(x, t)$ plane. That is, if $r(x, t)$ is a solution of equa tion (37) then $r[x-(u+c) t]$ is also a solution. The lines of slope $(c+u)$ in the $(x, t)$ plane are called characteristic curves.

Similarly to the above procedure we can multiply equation (34) by $-\frac{c}{p}$, and then add it to equation (33) yielding,

$$
-\frac{\partial \omega}{\partial t}+\frac{\partial u}{\partial t}-(c-u)\left(\frac{\partial u}{\partial x}-\frac{\partial \omega}{\partial x}\right)=0 \text {. }
$$

Letting $s=\frac{u-\omega}{2}$, and substituting in equation (38),

$$
\frac{\partial s}{\partial t}-(c-u) \frac{\partial s}{\partial x}=0,
$$

which is similar to equation (37) and which states that lines of constant a are propagated with velocity $u-c$ in the $(x, t)$ plane. The line 8 of slope $c+u$ and $u$ - c are called the characteristic and cross characteristic lines, respectively.

To illustrate the application of the method somewhat more in detail we recall that a linear combination $a \frac{\partial f}{\partial x}+b \frac{\partial f}{\partial y}$ of the two derivatives of a function $(x, y)$ means differentiation of $f$ along the direction $\frac{d x}{d y}=\frac{a}{b}$. We may then obtain $f$ by integration of the derivative of $f$ along this direction. This idea is then applied as follows:

Suppose we multiply equation (34) by $\lambda$ and add the result to equation (33). We have, then,

$$
\begin{aligned}
& \lambda \frac{\partial p}{\partial t}+\lambda u \frac{\partial p}{\partial x}+\lambda p \frac{\partial u}{\partial x}+\frac{\partial u}{\partial t}+u \frac{\partial u}{\partial x}+\frac{c^{2}}{\rho} \frac{\partial p}{\partial x}=0, \text { or } \\
& \lambda \frac{\partial p}{\partial t}+\left(\lambda u+\frac{c^{2}}{\rho}\right) \frac{\partial p}{\partial x}+\frac{\partial u}{\partial t}+(\lambda \rho+u) \frac{\partial u}{\partial x}=0,
\end{aligned}
$$

Note that this is in the form,

$$
a \frac{\partial p}{\partial t}+b \frac{\partial p}{\partial x}+c \frac{\partial u}{\partial t}+d \frac{\partial u}{\partial x}=0 \text {, }
$$


and if $\frac{a}{b}=\frac{c}{d}$ then the derivation of $p$ and $u$ are in the same direction.

This condition is satisfied if

$$
\begin{aligned}
& \lambda=\frac{\lambda u+\frac{c^{2}}{p}}{\lambda p+u}, \quad \text { or }, \\
& \lambda= \pm \frac{c}{p},
\end{aligned}
$$

$\lambda= \pm \frac{c}{\rho}$ is therefore an integrating factor and we have used it in obtaining, in conjunction with the Riemann invariant $\omega$, equations (37) and (39).

The direction determined by $\frac{a}{b}=\frac{c}{d}$ above is called characteristic and, in general, depends on the values of $x$ and $t a s$ well as $p$ and $u$ at the point.

Application of Method of Characteriatics to Isothe rmal Flow

Consider an isothermal flow in a perfect gas. For a perfect gas the velocity of sound, $c=c_{0}$, a constant, since $c=\sqrt{\frac{P}{p}}$ and $P \sim p T$, and if $\mathrm{T}=$ constant $\mathrm{c}=$ constant.

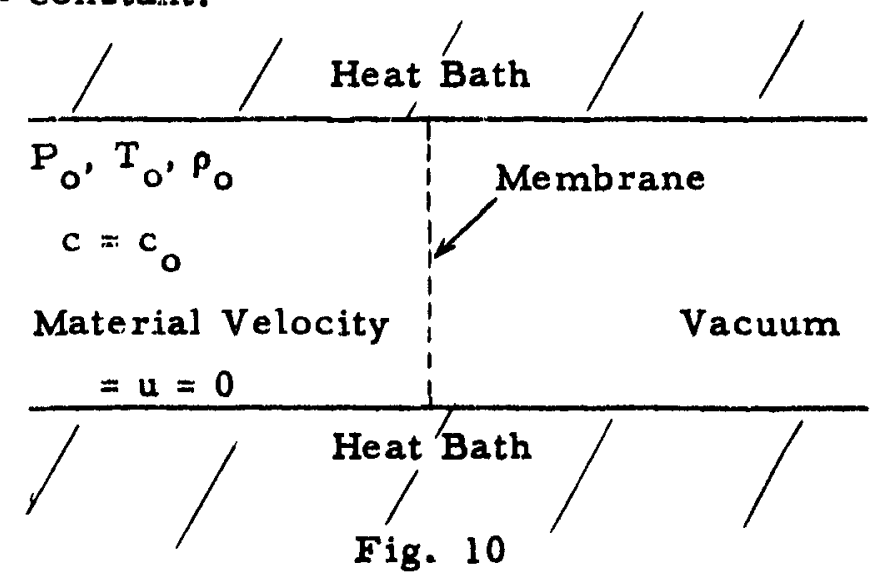

At $t=0$ the membrane above is boken and an isothermal expansion takes place. The flow pattern is such that the cross characteristic curves are straight lines and this is refered to as a "simple wave" (Courant-Friedrichs, p. 59).

Consider the behavior of the characteristic in the $(x, t)$ plane along which $\omega+u$ and $\omega-u$ are constant. The velocities of propagation of 
the se lines of constant $r$ or $s$ in the $(x, t)$ plane are $c+u$ and $u+c$, respectively, so that the slope of these lines is $\frac{1}{c+u}=$ constant and $\frac{1}{u-c}=$ constant, respectively, in the $(x, t)$ plane. Now at $t=0, c=c_{0^{\prime}} p=p_{0^{\prime}} \omega_{0}=0$, and $u=0$, therefore $\omega+u=0$, and $\omega-u=0$; and the slopes of the lines of constant $r$ and $s$ will be $\frac{1}{c_{o}}=$ constant, and $-\frac{1}{c_{0}}=$ constant, respectively.

We may, therefore, plot out the characteristic curves as in Fig. 11.

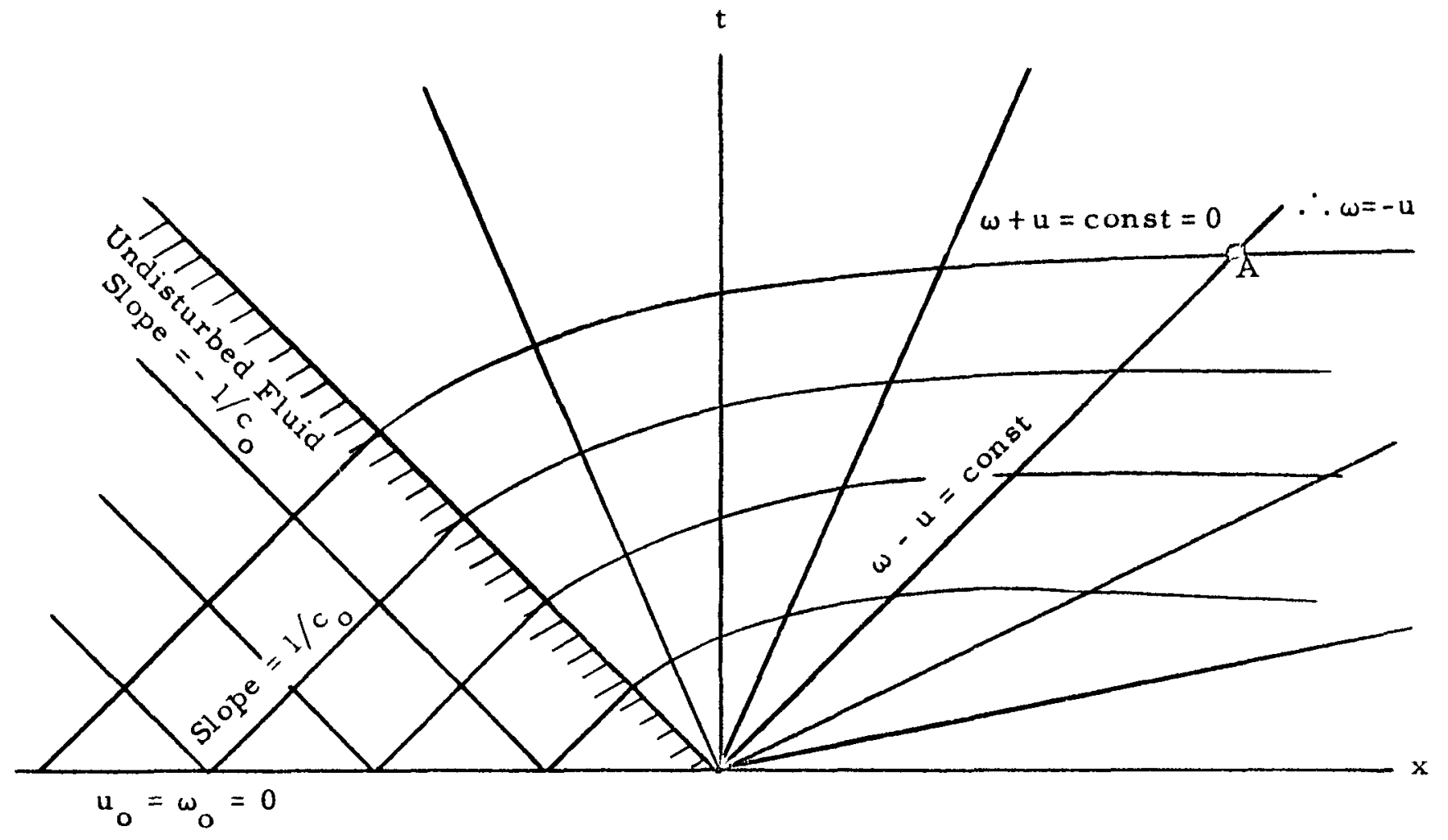

Fig. 11

At the point $A$ we have $\omega=-u$ and $\omega-u=$ const. Hence $\omega$ and $u$ are constant along the characteristic described by $\omega-u=$ const. Since we can express the sound speed as a function of $\omega$, we have $c=$ const along this characteristic. Hence this characteristic is a straight line of slope $\frac{1}{u-c}$. 
To the left of the characteristic of slope $-\frac{1}{c_{0}}$ and passing through the origin we have undisturbed fluid. This limiting characteristic describes a rarefaction wave moving into the undisturbed gas with speed $c_{0}$.

Now, for a cross characteristic, the velocity of propagation is

$$
\frac{d x}{d t}=u-c=\text { const or } \quad x=(u-c) t,
$$

for an isothermal expansion $\omega=c \ln \frac{p}{p_{0}}$,

but along a characteristic $\omega=-u$. Therefore, at a point of intersection between a characteristic and a croso-characteristic,

$$
x=-(\omega+u) t=-c t \ln \frac{p}{p_{0}}-c t
$$

or since $c=c_{0}$ in the undisturbed gas

$$
\frac{p}{p_{0}}=e^{-\left(\frac{x+c_{0} t}{c_{0} t}\right)}, \quad \frac{u}{c_{0}}=\frac{x+c_{0} t}{c_{0} t}
$$

for an isothermal blowoff.

The properties of an isothermal blowoff are shown in Fig. 12.

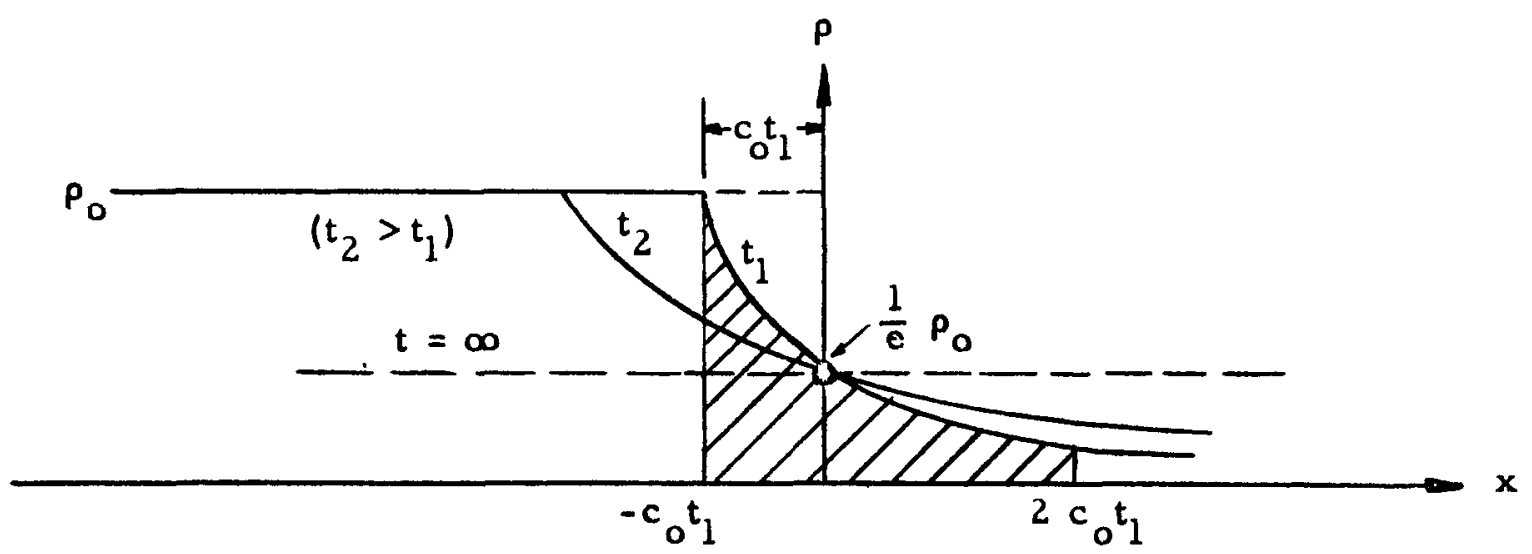

Fig. 12

The curve contains an apparent paradox in that the density curves for $t>0$ extend out to $+\infty$ so that the matter at far distances is moving very fast. However the density at large distances is very small. In fact, the ratio of matter included in the blowoff from $-c_{0} t$ to $+2 c_{0} t$ to that in the original slab of matter, or $\frac{1}{c_{0} t p_{0}} \int_{-c_{0} t}^{2 c_{0} t} \rho d x \approx 95$ percent. This can be seen 
from equation (42), for large $x, p$ is small but $u$ is large. A plot of $u$ is also shown in Fig. 13.

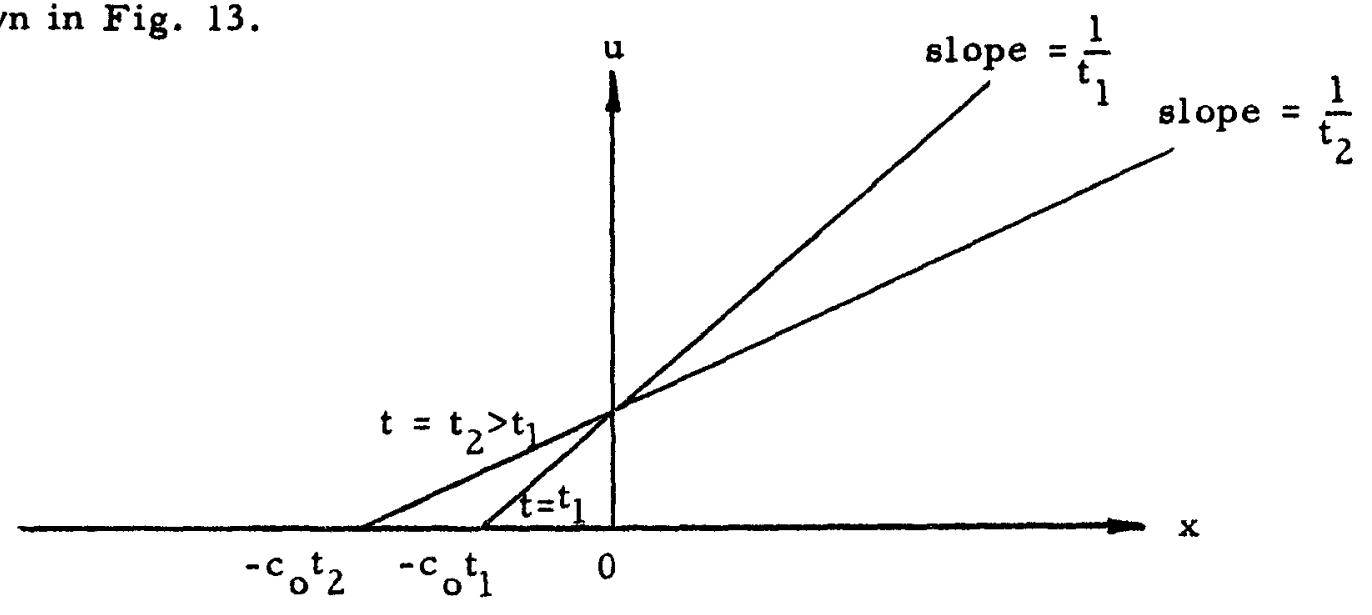

Fig. 13

There is a constant flux of matter across the interface at $x=0$ equal to $\frac{1}{e} p_{0} c_{0}$.

\section{Application of Method of Characteristics to an Adiabatic Expansion}

Consider an adiabatic expansion in the same geometry as used above. The characteristic curves again appear in Fig. 14.

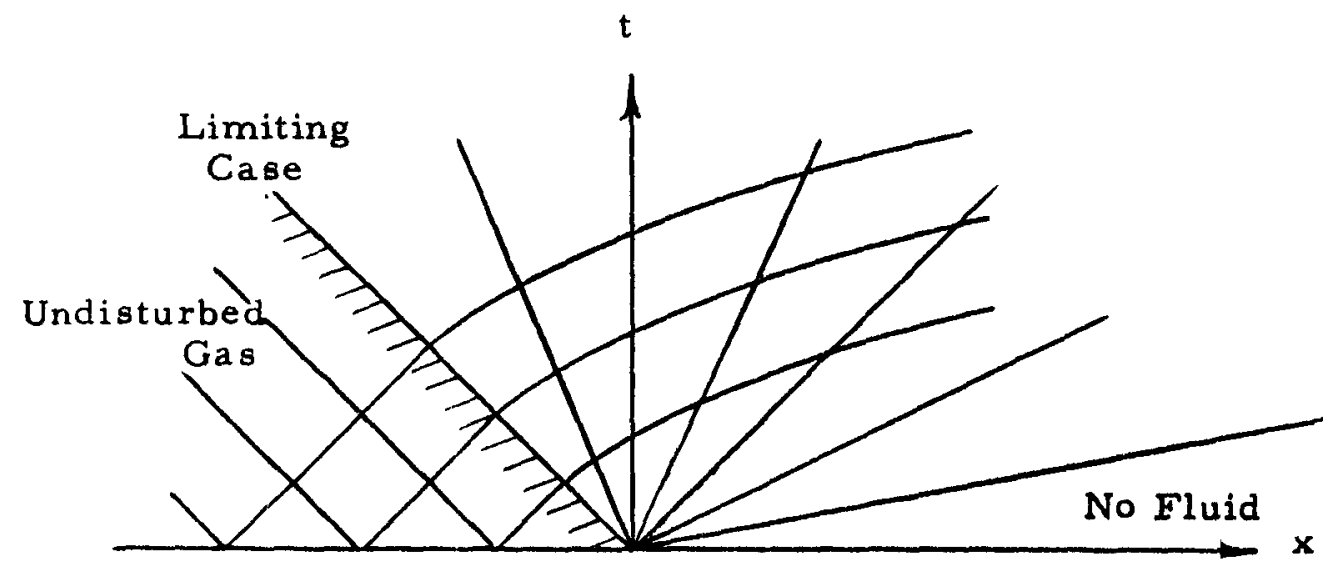

Fig. 14

By the same reasoning as for the isothermal case the cross characteristic lines are straight lines through the origin to the right of 
the limiting characteristics line.

$$
\text { Here } \omega=\int_{\rho_{0}}^{p} c(\rho) \frac{d \rho}{\rho}
$$

and

$$
\begin{aligned}
P & =P_{0}\left(\frac{\rho}{\rho_{0}}\right)^{\gamma} \\
c^{2} & =\frac{\partial P}{\partial \rho}=\left(\frac{P_{0} \gamma}{\rho_{0}}\right)\left(\frac{\rho}{\rho_{0}}\right)^{\gamma-1}=c_{0}^{2}\left(\frac{\rho}{\rho_{0}}\right)^{\gamma-1} \\
\therefore c & =c_{0}\left(\frac{\rho}{\rho_{0}}\right)^{\frac{\gamma-1}{2}} . \\
\text { Let } y & =\frac{\rho}{P_{0}}
\end{aligned}
$$

$$
\therefore \omega=c_{0} \int_{1}^{\frac{\rho}{\rho_{0}}} y^{\frac{\gamma-3}{2}} d y=\left[c_{0}\left(\frac{2}{\gamma-1}\right) y^{\frac{\gamma-1}{2}}\right]_{1}^{\frac{\rho}{\rho_{0}}} \text {. }
$$

$\therefore$ for a $y$-law

$$
\left.\begin{array}{l}
\omega=\frac{2}{\gamma-1} c_{0}\left[\left(\frac{\rho}{\rho_{0}}\right)^{\frac{\gamma-1}{2}}-1\right] \\
\omega=\frac{2}{\gamma-1} c_{0}\left(\frac{c}{c_{0}}-1\right) \\
\frac{c}{c_{0}}=\frac{\gamma-1}{2} \frac{\omega}{c_{0}}+1
\end{array}\right\}
$$

$$
\text { Now } \frac{d x}{d t}=u-c(\omega)=u-c_{0}\left[\frac{y-1}{2} \frac{\omega}{c_{0}}+1\right]
$$

or

$$
\begin{aligned}
& x=u t-c_{0}\left[\frac{L_{2}}{2} \frac{\omega}{c_{0}}+1\right] t \\
& \frac{x-u t}{c_{0} t}=-\left(\frac{1-1}{2} \frac{\omega}{c_{0}}+1\right)
\end{aligned}
$$

Along a characteristic $\omega=-u$. 
Therefore,

$$
\begin{aligned}
& x=-\left(\frac{y+1}{2}\right) \omega t-c_{0} t \\
& \omega=-\frac{\left(x+c_{0} t\right)}{\frac{y+1}{2} t}=\frac{2}{\gamma-1} c_{0}\left[\left(\frac{p}{\rho_{0}}\right)^{\frac{y-1}{2}}-1\right]
\end{aligned}
$$

or $\quad \frac{p}{p_{0}}=\left[1-\left(\frac{\gamma-1}{\gamma+1}\right)\left(\frac{x+c_{0} t}{c_{0} t}\right)\right]^{\frac{2}{\gamma-1}}$.

Note that $p=0$ when $x+c_{0} t=\frac{\gamma+1}{\gamma-1} c_{0} t$ or $x=\frac{2}{\gamma-1} c_{0} t$.

Therefore, the front of the wave has a finite velocity equal to $\frac{2}{\gamma-1} c_{0}$, whereas in the isothermal case the velocity approached infinity as $x$ approached infinity.

The curves of density and velocity are given in Fig. 15.

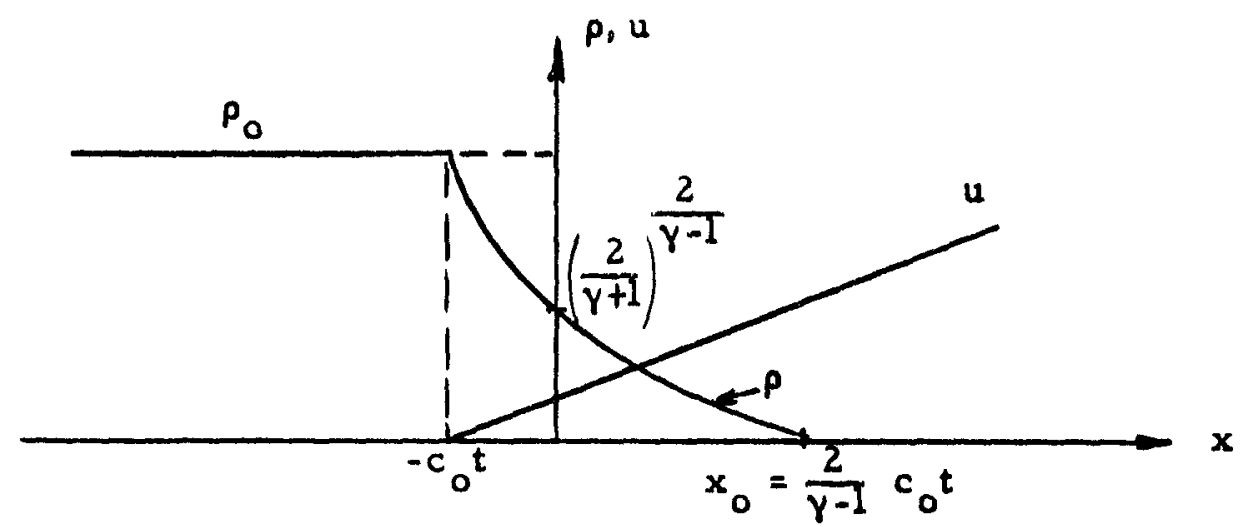

Fig. 15

For $y-2$ the matter front moves twice as fast forward as the rariaction proceeds back.

As $\gamma$ approaches one we approach the isothermal case, for

$$
\lim _{N \rightarrow \infty}\left(1+\frac{Z}{N}\right)^{N} \cdot e^{-N} \text { or } \lim _{x \rightarrow 1}\left(\frac{\rho}{\rho_{0}}\right) \text { adiabatic }=e^{-\left(\frac{x+c_{0} t}{c_{0} t}\right)}=\left(\frac{p}{\rho_{0}}\right)_{\text {isothermal }} \text {. }
$$


The velocity of matter flow for the adiabatic case is,

$$
\frac{u}{c_{0}}=\frac{2}{y+1}\left(\frac{x+c_{0} t}{c_{0} t}\right)
$$

and at $x=0$

$$
u=\frac{2}{\gamma+1}
$$

The vacuum approximation used above is useful for cases in which we have a heavy medium expanding into a very light medium. 


\section{UNIVERSITY OF CALIFORNIA, RADIATION LABORATORY LIVERMORE SITE}

LECTURES ON HYDRODYNAMICS AND SHOCK WAVES

Lectures by Robert Lelevier - Notes by Boris Ragent

April 30,1954

\section{Lecture 5: APPLICATION OF THE METHOD OF THE RIEMANN INVARIANT TO CALCULATION OF SHOCKS MOVING FROM HEAVY INTO LIGHT MEDIA}

In the last lecture we introduced the notion of the Riemann invariant, $\omega=\int_{\rho_{0}}^{p} c(p) \frac{d p}{p}$, and its application to the calculation of simple waves, i.e. motion in the $(x, t)$ plane which is characterized by the fact that the cross characteristics are straight lines. We now apply these notions to the calculation of shocks proceeding from a heavy to a light medium.

Conside $r$ the one-dimensional situation shown in Fig. 16. This is the typical situation as it occurs in shock tubes.

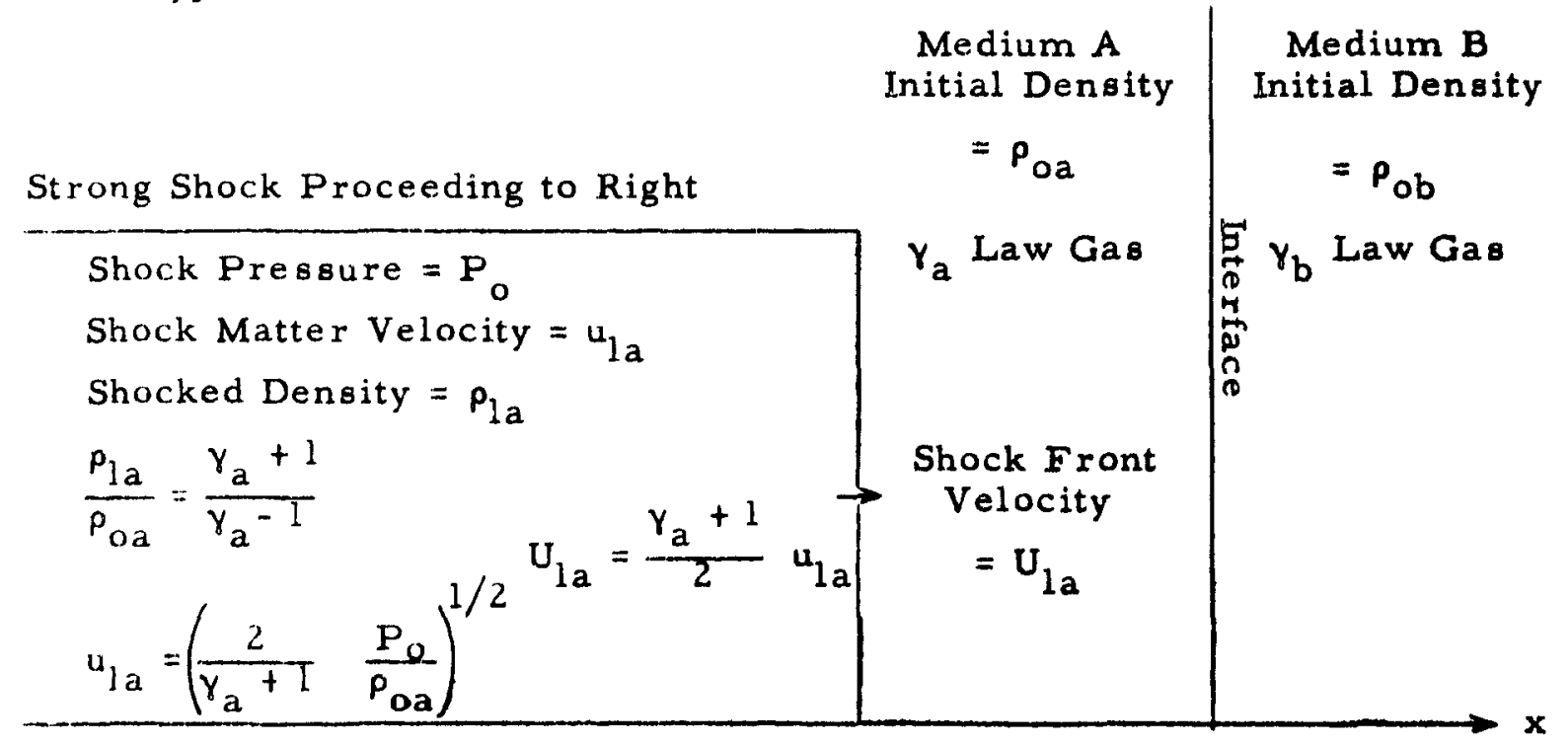

Fig. 16. Shock Approaching Interface 
Assume $P_{\text {ob }}<P_{\text {oa }}$. The shock moves to the interface at which time a shock is sent into medium $B$ and a rarefaction fan move $s$ back into medium A. At some later time the situation is shown in Fig. 17.

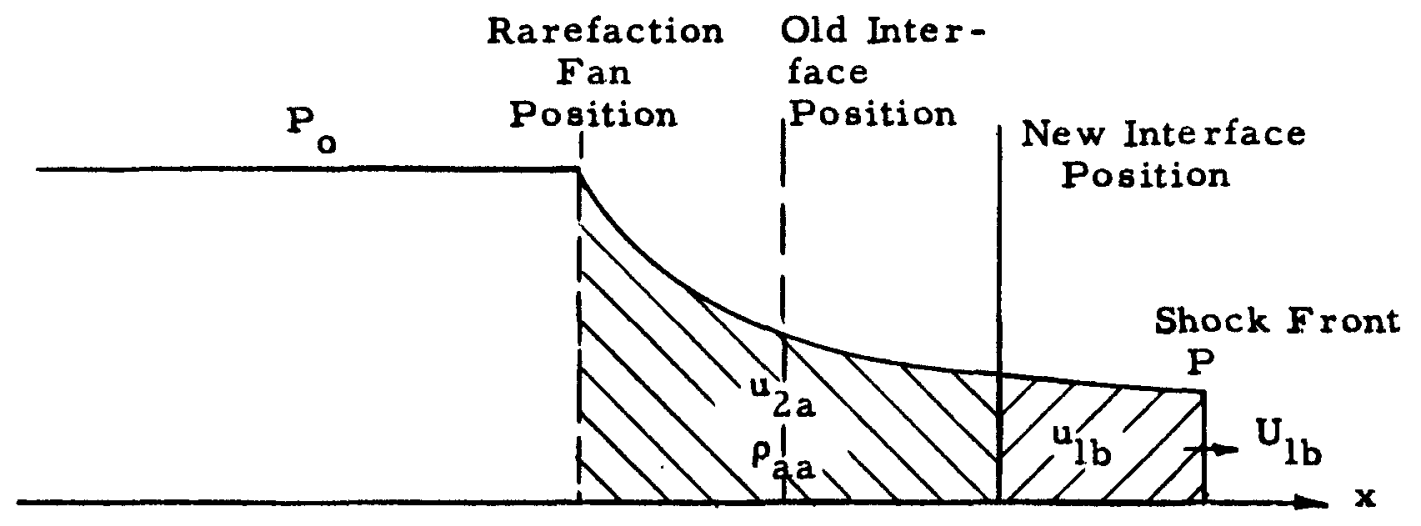

Fig. 17 Shock Progression into Medium B

Now, for a simple wave $\omega+\mathrm{u}=\mathrm{K}$, a constant. If we follow the lines of constant $\mathrm{K}$ back into the medium $\mathrm{A}$ at $\mathrm{t}=0$ we find that $\omega=0$, but $u=u_{1 a}$ or, $u=u_{1 a}-\omega_{\text {, }}$ or if we sperifically solve for the matter velocity in the rarefaction fan,

$$
u_{2 a}=u_{1 a}-w
$$

Now, previously we saw that for an adiabatic expansion,

$$
\begin{aligned}
& \omega=\frac{2}{\gamma_{a}-1} c_{a}\left[\left(\frac{P}{P_{0}}\right)^{\frac{\gamma_{a}-1}{2 \gamma_{a}}}-1\right] \\
& c_{a}=\left(\frac{\gamma_{a} P_{0}}{\rho_{1 a}}\right)^{1 / 2}=\text { sound speed in medium } A .
\end{aligned}
$$

We also know that for a shock,

$$
u_{1 b}=\left(\frac{2}{\gamma_{b}+I} \frac{P}{\rho_{o b}}\right)^{1 / 2},
$$

the requirement of continuity of flow across the interface leads to one further requirement.

$$
u_{1 b}=u_{2 b}
$$


From equations (52), (53), and (54) we may solve for P. Letting $y=\frac{P}{P_{0}}<1, \quad x=\frac{P_{o a}}{P_{o b}}>1$, and remembering that $u_{1 a}=\left(\frac{2}{x_{a}+I} \frac{P_{0}}{P_{o a}}\right)^{1 / 2}$, we obtain the transcendental equation for $y$,

$$
1+\left(\frac{2 \gamma_{a}}{\gamma_{a}-1}\right)^{1 / 2}\left(1-y^{\frac{\gamma_{a}-1}{2 \gamma_{a}}}\right)=\left(\frac{\gamma_{a}+1}{\gamma_{b}+1}\right)^{1 / 2} x^{1 / 2} y^{1 / 2} .
$$

Consider a typical graphical solution of equation (55) as 1llustrated in Figure 818 and 19 .

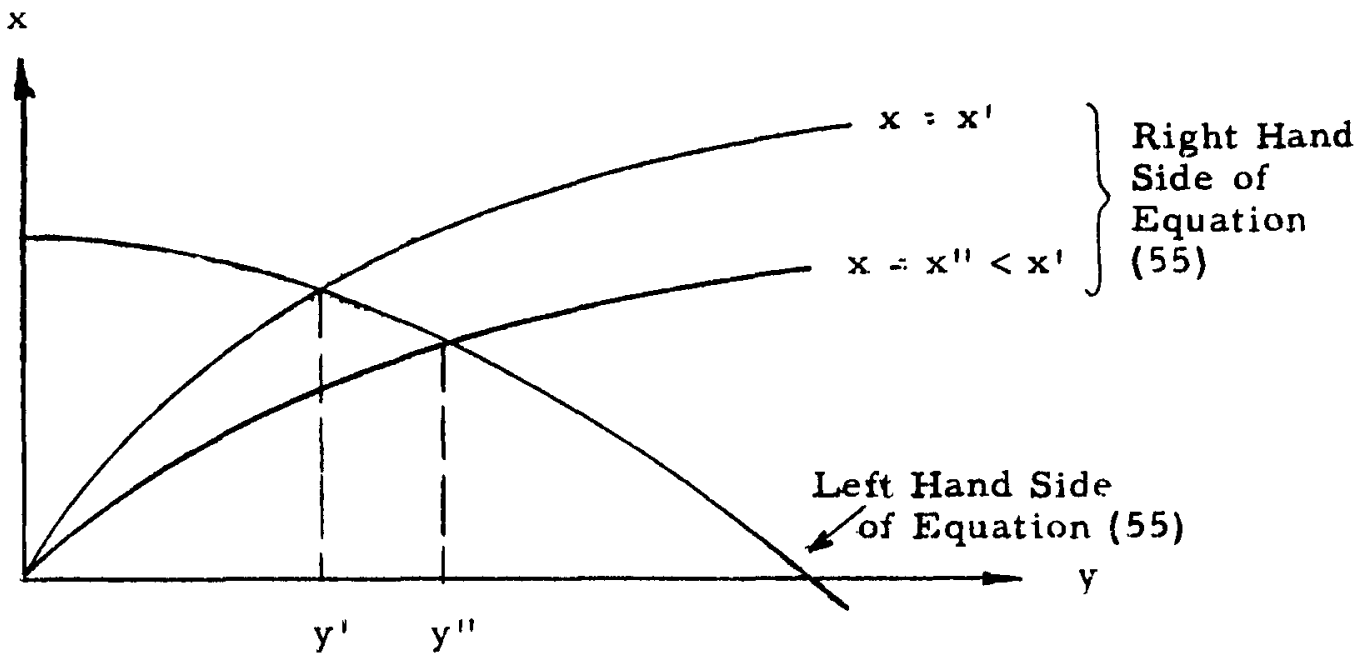

Fig. 18 Graphical Solution of Equation (55) with $\gamma_{a}$ and $\gamma_{b}$ Fixed

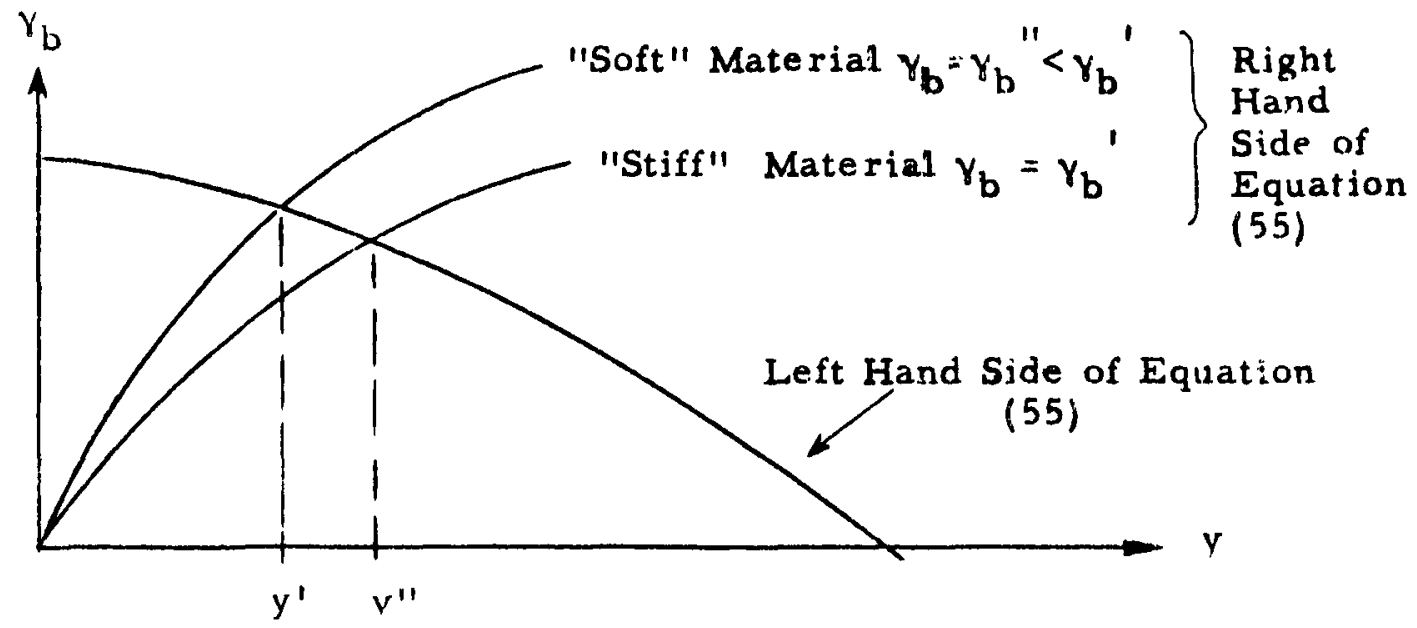

Fig. 19 Graphical Solution of Equation (55) with $x$ and $\gamma_{a}$ Fixed 
Figures 18 and 19 show that the stiffest material (greatest $\gamma$ ), and the smallest density mismatch give rise to the greatest shock pressure ratio, or allow the shock to progress with minimum pressure attenuation. The dependence of $y$ on $\gamma$ is, however, much smaller than its dependence on $x$, the density ratio.

\section{Illustrative Example:}

In order to get some idea of the orders of magnitude involved here we will substitute some typical numbers into equation (55). For ease of calculation let us suppose that $\gamma_{\mathrm{a}}=2$.

Then we obtain for equation (55),

$$
y^{1 / 4}=\frac{-1 \pm\left[1+3\left(\frac{3 x}{\gamma_{b}+1}\right)^{1 / 2}\right]^{1 / 2}}{\left(\frac{3 x}{\gamma_{b}+1}\right)^{1 / 2}} .
$$

We know that $y$ must be positive and less than one (except in the case for $x>1$, in which case we use the reflection theory of the firat part of the last lecture). Therefore we must use the positive sign in equation (56).

Table II gives $y$ as a function of $x$ for the case $y=2$.

Table II. SHOCK PRESSURE RATIOS AS A FUNCTION OF DENSITY

$$
\text { MISMATCH }
$$

\begin{tabular}{r|l}
$\mathbf{x}$ & $\mathbf{y}$ \\
\hline 1 & 1 \\
4 & 0.4 \\
9 & 0.25 \\
25 & 0.13 \\
100 & 0.04
\end{tabular}

Note that the $\gamma^{\prime}$ 's used in the text above are not, in general, the ratios of the specific heats of the gas. In general, we define the $\gamma^{\prime}$ from an empirical Hugoniot relation, using equation (26) of Lecture 3. Another way of defining $\gamma$ is from $c^{2}=\left(\frac{\partial P}{\partial \rho}\right)_{s}$. For a non $\gamma$-law gas, we must determine the value 
of $Y$ at each value of $p$ and $P$ and $u s e$ it in an iterative process in equation (55) above. 
UNIVERSITY OF CALIFORNIA, RADIATION LABORATORY

LIVERMORE SITE

\section{LECTURES ON HYDRODYNAMICS AND SHOCK WAVES}

Lectures by Robert Lelevier - Notes by Boris Ragent

May 7, 1954

Lecture 6: NUMERICAL TECHNIQUES FOR SOLVING DIFFERENTIAL EQUATIONS. THE FINITE DIFFERENCE METHOD

The problem to be discussed in this lecture is that of putting the differential equations with which we have been dealing into a form suitable for numerical calculation, particularly for use with modern digital type computing machines. Our technique for a given function, $f(t)$, will be to sample $f(t)$ at discrete points, determine the slope of $f(t)$ at these points, and attempt to fit a curve through any one or more of the se discrete points. In general, through any two, three, four, etc., points we can fit respectively a straight line, parabola, cubic, etc. General. usage is to calculate $f(t), f^{\prime}(t)$ and $f^{\prime \prime}(t)$, at a discrete point to second order in the finite difference intervals between discrete points.

In Fig. $20 \mathrm{f}(\mathrm{t})$ is represented schematically. $f\left(t_{0}\right), f\left(t_{1}\right)$ and $f\left(t_{2}\right)$ are the discrete points

$r(t)$

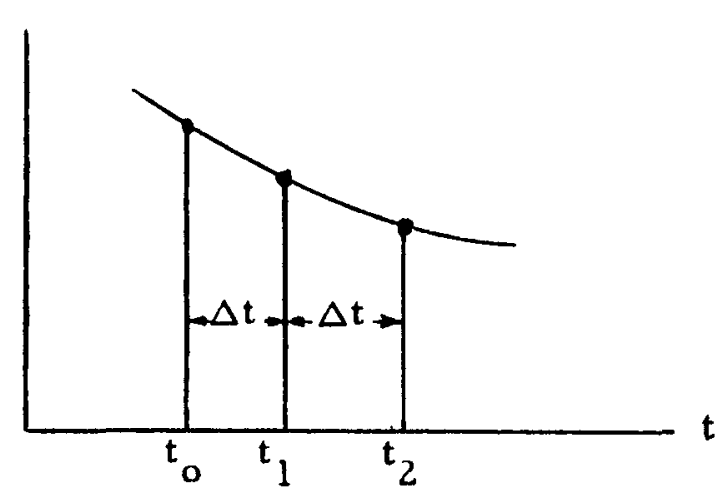

Fig. 20 
and $t_{2}-t_{1}=t_{1}-t_{0}=\Delta t$ is the difference interval.

We then represent, to second order in $\Delta t$ the slope of $f(t)$ by,

$$
\left.f^{\prime}(t)\right]_{t_{2}}=t_{1}=\frac{f\left(t_{2}\right)-f\left(t_{o}\right)}{2 \Delta t} .
$$

This is correct to second order, since if we expand $f\left(t_{1}\right)$ in a Taylor's series,

$$
\begin{aligned}
& f\left(t_{2}\right)=f\left(t_{1}\right)+f^{\prime}\left(t_{1}\right) \Delta t+f^{\prime \prime} \frac{\left(t_{1}\right)(\Delta t)^{2}}{2 !}+0(\Delta t)^{3} \\
& f\left(t_{0}\right)=f\left(t_{1}\right)-f^{\prime}\left(t_{1}\right) \Delta t+f^{\prime \prime}\left(t_{1}\right) \frac{(\Delta t)^{2}}{2 !}-0(\Delta t)^{3}
\end{aligned}
$$

and $\frac{f\left(t_{2}\right)-f\left(t_{2}\right)}{2 \Delta t}=f^{\prime}(t)$ to second order in $\Delta t$.

Similarly,

$$
\left.f^{\prime \prime}(t)\right]_{t=t_{1}}=\frac{f\left(t_{2}\right)-2 f\left(t_{1}\right)+f\left(t_{0}\right)}{(\Delta t)^{2}}
$$

correct to second order in $\Delta t$.

There are two general criteria which must be investigated before proceeding with any proposed scheme of numerical calculation. These are:

1. Accuracy. Does the scheme give a solution which converges to the solution of the differential equation as $\Delta t \rightarrow 0$ ?

2. Stability. Do small errors tend to grow or damp out dur. ing the numerical calculations.

To illustrate the technique involved let us consider an example of an ordinary differential equation, and calculate the accuracy and stability cri.. teria for several proposed computational methods.

Consider the equation,

$$
\frac{d f(t)}{d t}=-\gamma f(t) .
$$

Suppose we know $f(t)$ up to some time $t=t_{1}$, and wish to know it at $t=t_{2}$. One can fit a parabola at three points, yielding the derivative at 
$t=t_{2}$

$$
\left(\frac{d f}{d t}\right)_{t=t_{2}}=\frac{\frac{3}{2} f\left(t_{2}\right)-2 f\left(t_{1}\right)+\frac{1}{2} f\left(t_{0}\right)}{\Delta t},
$$

correct to second order in $\Delta t$. Using the notation $t_{1}=n \Delta t_{2} t_{0}=(n-1) \Delta t$, $t_{2}=(n+1) \Delta t, f\left(t_{1}\right)=f(n)$ where $n$ indicates the $n$th point, in this case the $t_{1}$ point, we have from equations (59) and (60)

$$
\begin{aligned}
& \quad \frac{3}{2} f(n+1)-2 f(n)+\frac{1}{2} f(n-1)=-\gamma \Delta t f(n+1) \\
& \text { or, } \quad\left(\frac{3}{2}+\gamma \Delta t\right) f(n+1)-2 f(n)+\frac{1}{2} f(n-1)=0,
\end{aligned}
$$

which is the finite difference equation expressing $f(n+1)$ in terms of $f(n)$ and $f(n-1)$.

Another more natural way of obtaining the difference equation would be to use one of the following choices.

$$
\left(\frac{d f}{d t}\right)_{n \Delta t}=\frac{f(n+1)-f(n)}{\Delta t} .
$$

From equation (59) we have three choices

$$
\frac{f(n+1)-f(n)}{\Delta t}=-\gamma\left\{\begin{array}{l}
f(n) \\
f(n+1) \\
\frac{1}{2}[f(n+1)+f(n)]
\end{array}\right\}
$$

yielding the three possible difference equations

$$
\begin{aligned}
& f(n+1)=f(n)(1-y \Delta t) \\
& f(n+1)=f(n)(1+y \Delta t) \\
& f(n+1)=f(n)\left(\frac{1-\frac{\gamma}{2} \Delta t}{1+\frac{\gamma}{2} \Delta t}\right) .
\end{aligned}
$$

Equations (61), (62), (63), and (64) represent four different attempts to write the difference equation. In order to make a choice we must test each alternative for accuracy and stability. 
Consider the case of equation (61). Assume that $f(n) \sim \frac{1}{\xi^{n}} \cdot$ Then from equation (61) we have,

$$
\frac{3}{2}+\gamma \Delta t \frac{1}{\xi}-2+\frac{1}{2} \xi=0
$$

or $\quad \xi=2 \pm(L-2 \psi \Delta t)^{1 / 2}$.

Here we have two roots. Since the difference equation is linear any linear combination of the two roots will be a solution. Therefore,

$$
f(n)=\frac{A}{(2+\sqrt{1-2 \gamma \Delta t})^{n}}+\frac{B}{(2-\sqrt{1-2 \gamma \Delta t})^{n}} .
$$

In order to have a real solution we must have

$$
\begin{aligned}
& 2 y \Delta t<1, \\
& \text { or } \quad \Delta t<\frac{1}{2 \gamma},
\end{aligned}
$$

which is the stability condition on equation (61). Now, suppose that a $\mathbf{n} \rightarrow \infty$, $\Delta t \rightarrow 0$ such that $t=n \Delta t$. Then considering the second term in equation (67) and expanding,

$$
2-\sqrt{1-2 \gamma \Delta t} \rightarrow 2-(1-\gamma \Delta t)=1+\gamma \Delta t
$$

and

$$
\begin{aligned}
(1+y \Delta t)^{n}=\left(1+\frac{Y t}{n}\right)^{n} \rightarrow e^{y t} \text { as } n & \rightarrow \infty \\
\Delta t & \rightarrow 0
\end{aligned}
$$

or the right hand side of equation (67) becomes

$$
\begin{aligned}
& \lim f(n)=B e^{-\gamma t} \text {, or the right hand } \\
& {\left[\begin{array}{rl}
\Delta t & \rightarrow 0 \\
n & \rightarrow \infty \\
n \Delta t & \rightarrow t
\end{array}\right] }
\end{aligned}
$$

term converges to the correct solution in the limit. Now the left hand side of equation (67) as $n \rightarrow \infty$ becomes,

$$
2+1-\gamma \Delta t=3\left(1-\gamma \frac{\Delta t}{3}\right)
$$


and

$$
3^{n}\left(1-y \frac{\Delta t}{3}\right)^{n} \rightarrow 3^{n} e^{-\frac{y t}{3}}
$$

or left hand side of equation (67) goes to $A \frac{e^{\frac{y_{t}}{3}}}{3^{n}}$, which represents a transient which damps as $n \rightarrow \infty$. This method of differencing does not appoar to be particularly good even though it is accurate to second order in the differences.

Consider equation (64). Assume here that $f(n) \sim \xi^{n}$.

$$
\begin{aligned}
& \therefore \xi=\frac{1-\frac{Y}{2} \Delta t}{1+\frac{Y}{2} \Delta t} \quad \text { from equation (64) } \\
& f(n)=\left[\frac{1-\frac{Y}{2} \Delta t}{1+\frac{Y}{2} \Delta t}\right]^{n}
\end{aligned}
$$

The requirement for positive roots (assuming a positive behavior for the roots of equation (59)) is

$$
\frac{y}{2} \Delta t<1
$$

$$
\text { or } \Delta t<\frac{2}{\mathrm{Y}},
$$

which is the stability requirement for equation (64).

The accuracy is again determined by expanding,

$$
\begin{aligned}
& f(n)=\left[\frac{1-\frac{y}{2} \Delta t}{1+\frac{y}{2} \Delta t}\right]^{n} \sim\left[1-\frac{n}{2} y \Delta t+n \frac{(n-1)}{2} \frac{y^{2}}{4}(\Delta t)^{2}+0(\Delta t)^{3}\right] . \\
& {\left[1-\frac{n y}{2} \Delta t+\frac{n(n+1)}{2} \frac{y^{2}}{4} \Delta t+0\left(\Delta t^{3}\right)\right]} \\
& \sim 1-n \Delta t+\frac{n^{2} y^{2}(\Delta t)^{2}}{2}+0\left(\Delta t^{3}\right)
\end{aligned}
$$




$$
\begin{aligned}
& =1-\gamma t+\frac{\gamma^{2} t^{2}}{2}+0\left(\Delta t^{3}\right) \\
& =e^{-\gamma t}+0\left(\Delta t^{3}\right)
\end{aligned}
$$

or the correct solution to second order, without the transient term we obtained by using equation (61).

Consider equation (62), (forward differencing).

Here

$$
\begin{aligned}
f(n) & \sim(1-\gamma \Delta t)^{n} \\
& \sim 1-\gamma n \Delta t+\frac{n(n-1)}{2} \gamma^{2}(\Delta t)^{2} \\
& \sim 1-\gamma t+\frac{\gamma^{2} t^{2}}{2}-\frac{(\gamma t)(\gamma \Delta t)}{2}+\ldots \\
& =e^{-\gamma t}+0\left(\Delta t^{2}\right)
\end{aligned}
$$

or this equation does not yield a solution which converges to $f(t)$ to the second order. Note also that the stability requirement for real solutions is $\Delta t<\frac{1}{\gamma}$. This method is not as accurate as that of equation (61) or (64) above.

Consider equation (63), (backward differencing).

Here $f(n) \sim(1+\gamma \Delta t)^{-n}$. Here we do not have a stability condition, that is, the solution is "unconditionally" stable for any positive $\Delta t$. On the other hand, we also have only first order accuracy here. In order to obtain any accuracy at all we would have to impose the condition $\gamma \Delta t \ll 1$, since this equation is actually only good to first order.

The best method of differencing therefore appears to be that of equation (64). 


\title{
UNIVERSITY OF CALIFORNIA, RADIATION LABORATORY
}

\author{
LIVERMORE SITE
}

\section{LECTURES ON HYDRODYNAMICS AND SHOCK WAVES}

\author{
Lectures by Robert Lelevier - Notes by Boris Ragent
}

\author{
May 14, 1954 \\ Lecture 7: NUMERICAL SOLUTIONS OF PARTIAL DIFFERENTIAL \\ EQUATIONS
}

In this lecture we shall continue the discussion of differencing of differential equations. In general, each differencing scheme for a partial differential equation must be tested for accuracy and stability. In order to illustrate the typical technique involved, difference equations for an important type of partial differential equation arising in hydrodynamics will be constructed and tested for stability and accuracy.

Consider the equation,

$$
\frac{\partial f(x, t)}{\partial t}+u \frac{\partial f(x, t)}{\partial x}=0
$$

where $u$ is a constant. In the $(x, t)$ plane construct a grid as shown in Fig. 21. Suppose that we know the initial conditions on $f(x, t)$ at some time $t=n \Delta t$ for all

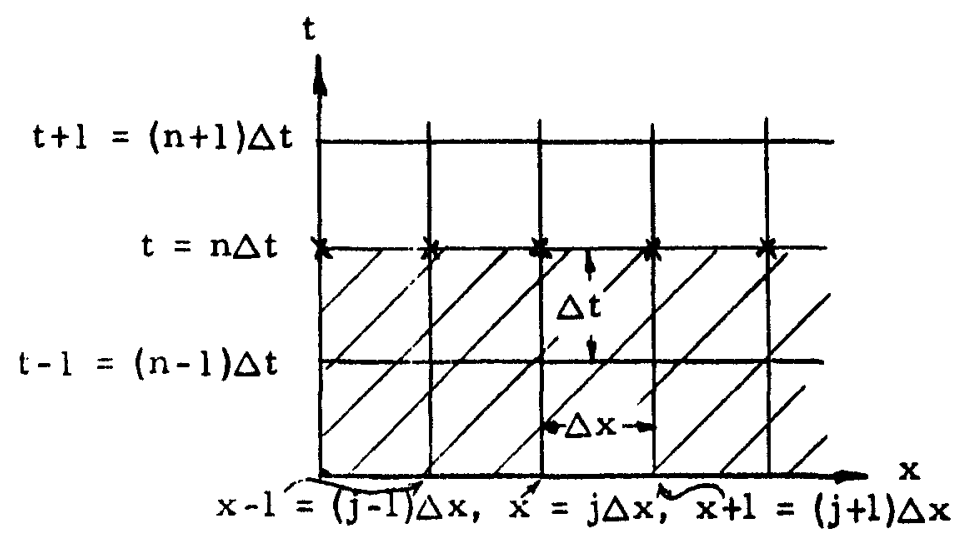

Fig. 21 
values of $x$, as indicated by the crosses in Fig. 21. Extending the notation of the previous lecture we characterize the value of $f(x, t)$ at $(x=j \Delta x, t=n \Delta t)$ by the symbol $f_{j}{ }^{n}$.

Our problem will be to calculate the values of $f(x, t)$ for value $s$ of $t$ greater than $n \Delta t$. There are several possible methods of proceeding, involving a choice of differencing methods with which to move across the grid. Each of the se methods must be tested for stability and accuracy, as in the example in the last lecture for an ordinary differential equation, in order to determine which of the conceivable methods is practical.

In equation (71), for example, we will wish to calculate $\frac{\partial f}{\partial t}$ and $\frac{\partial f}{\partial x}$ correct to second order at the points of the grid in question. We might attempt to set up the symmetric derivatives, centered at the point $(j, n)$, correct to second order, as follows:

$$
\begin{aligned}
& \left.\frac{\partial f}{\partial t}\right]_{x}=\frac{f_{j}^{n+1}-f_{j}^{n-1}}{2 \Delta t} \\
& \left.\frac{\partial f}{\partial x}\right]_{t}=\frac{f_{j+1}^{n}-f_{j}-1^{n}}{2 \Delta x}
\end{aligned}
$$

Then substituting into equation (71),

$$
\frac{f_{j}^{n+1} \cdot f_{j}^{n-1}}{2 \Delta t}+u\left(\frac{f_{j+1}^{n}-f_{j-1}^{n}}{2 \Delta x}\right)=0
$$

This will enable us to solve for $f_{j}{ }^{n+1}$ since all of the other values in equation (74) are preaumed to be known. Notice that in equation (74) the value of $f_{j}{ }^{n}$ does not enter but that $f_{j}{ }^{n+1}$ instead is coupled to the three other pointe surrounding $f_{j}^{n}$. In cloing this calculation, therefore, we would have to store data for the values rif $f_{j}-1, f_{j}^{n-1}$, and $f_{j}+1$. Also note that the value of $f_{j}{ }^{n}$ does not affect $t$ l e derivative, and would only appear in calculating the values of the succeeding points in the calculation. This method of differencing, 
therefore, although possible is not very practical due to the large memory storage required initially, and the length of time the information must be stored.

Stability Test, Von Neumann Method

Assume that at the time $t=n \Delta t$ the true solution to the equation is $(0) f_{j}^{n}$. Suppose that the calculated value at $f_{j}^{n}$ is somewhat in error, so that

$$
\mathbf{f}_{j}^{n}=(0) f_{j}^{n}+\Delta f_{j}^{n}
$$

In order to investigate the propagation of this error, we substitute equation (75) into the original difference equation in order to get the first order variation equation (76) of the difference equation (74),

$$
\frac{\Delta f_{j}^{n+1}-\Delta f_{j}^{n-1}}{2 \Delta t}+u \frac{\left(\Delta f_{j+1}^{n}-\Delta f_{j}-1^{n}\right)}{2 \Delta x}=0
$$

Let us expand the perturbation in a Fourier series so that,

$$
\Delta f_{j}^{n}=\Sigma A_{k} e^{a_{k} n \Delta t} e^{i k j \Delta x}
$$

Let

$$
\xi_{k}=e^{a_{k} \Delta t}
$$

Then, introducing equation (77) into equation (76), and examining a given Fourier component, we arrive at

$$
\frac{\xi-\xi^{-1}}{\Delta t}+u\left(\frac{e^{i k \Delta x}-e^{-i k \Delta x}}{\Delta x}\right)=0
$$

For stability $/ \xi / \leqq 1$, or $a_{k} \leqq 0$ where $a_{k}$ is real.

Let $\gamma=u \frac{\Delta t}{\Delta x}$. Then equation (77) becomes

$$
\xi-\xi^{-1}+21 \gamma \sin k \Delta x=0 \text {, }
$$

or

$$
\xi=-i y \sin k \Delta x \pm\left(y^{2} \sin ^{2} k \Delta x+1\right)^{1 / 2}
$$




$$
\begin{aligned}
& \text { If } \gamma^{2} \leqq 1, \text { then }\left(\gamma^{2} \sin ^{2} k \Delta x+1\right)^{1 / 2} \text { is real. } \\
& / \xi /^{2}=1-\gamma^{2} \sin ^{2} k \Delta x+\gamma^{2} \sin ^{2} k \Delta x=1
\end{aligned}
$$

or the stability condition on equation (71) is,

$$
/ \mathrm{y} /=/ \mathrm{u} \frac{\Delta \mathrm{t}}{\Delta \mathrm{x}} / \leqq 1
$$

With this stability condition any error introduced neither grows nor damps.

\section{Altérnate Differencing Schemes}

Forward Differencing:

In this method we take a one-sided time derivative. Thus we have

$$
\left.\frac{\partial f}{\partial t}\right]_{x}=\frac{f_{j}^{n+1}-f_{j}^{n}}{\Delta t}
$$

Then substituting into equation (71) we obtain,

$$
\frac{f_{j}^{n+1}-f_{j}^{n}}{\Delta t}+u\left(\frac{f_{j+1}{ }^{n}-f_{j}-1^{n}}{2 \Delta x}\right)=0 \text {. }
$$

The stability condition, calculated by the Von Neumann method

becomes,

$$
\xi-1+\left(\frac{x}{2}\right)(2 i) \sin k \Delta x=0
$$

or,

$$
\begin{aligned}
& \xi=1-i y \sin k \Delta x \\
& / \xi /^{2}=1+y^{2} \sin ^{2} k \Delta x
\end{aligned}
$$

Since $\gamma$ is real, oin ${ }^{2} k \Delta x$ is real, and

$$
\xi \geq 1
$$

Therefore, in this echeme errore will grow exponentially, or the difference equation is unatable.

Non-Centered Differences:

Suppose that $u$ is positive so that the material velocity is in the direction of increasing $x$ and $t$. Then wo can write our difference equation 
as a first order equation, for $u>0$

$$
\frac{\mathbf{f}_{\mathbf{j}}^{\mathbf{n}+1}-\mathbf{f}_{\mathbf{j}}^{\mathbf{n}}}{\Delta t}+u\left(\frac{\mathbf{f}_{\mathbf{j}}^{\mathbf{n}}-\mathbf{f}_{\mathbf{j}-1}{ }^{\mathbf{n}}}{\Delta x}\right)=0 .
$$

Note if $u<0$ we would write

$$
\left.\frac{f_{j}^{n+1}-f_{j}^{n}}{\Delta t}+u\left(\frac{f_{j}+1^{n}-f_{j}^{n}}{\Delta x}\right)=0 .\right]
$$

Since this is only a first order equation we must use a fine grid to achieve any accuracy. Now, using the Von Neumann method, the stability equation reads,

$$
\xi-1+\gamma\left(1-e^{-i \frac{k}{2} \Delta x}\right)=0
$$

or

$$
\xi-1+i 2 \gamma e^{-i \frac{k}{2} \Delta x} \sin \frac{k}{2} \Delta x=0
$$

or

$$
\xi=1-i 2 \gamma e^{-i \frac{k}{2} \Delta x} \sin \frac{k}{2} \Delta x
$$

Consider a plot of $\xi$ in the imaginary plane, Fig. 22.

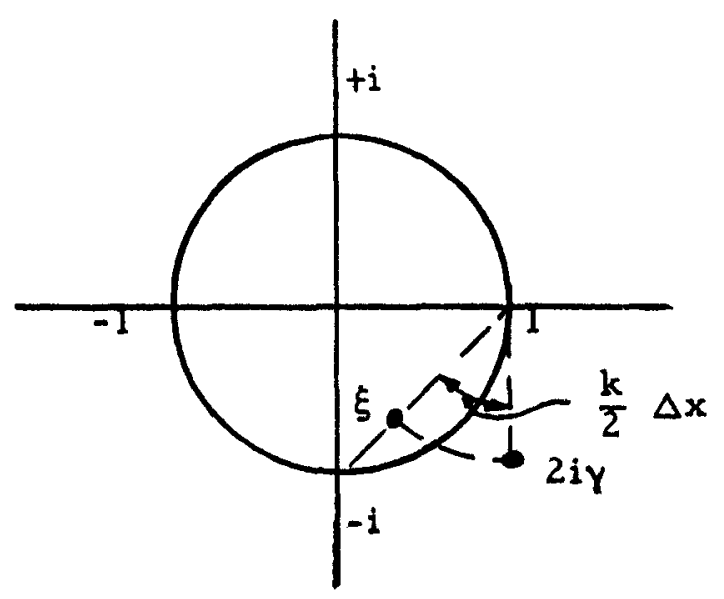

Fig. 22

For stability $/ \xi / \leqq 1$ so that for the worst case, $\frac{k}{2} \Delta x=\frac{\pi}{2}$,

a perturbation In resonance with the grid,

$$
\xi=1-2 \gamma, \text { or, }
$$




$$
0 \leq Y \leq 1
$$

is the condition for atability.

For $\gamma=1$, a non-practical case, an interesting situation arises. Consider the case of flow pictured in Fig. 23. If $Y=u \frac{\Delta t}{x}$ and if $\gamma=1$, then in one time step the flow jumps from $t=0$ to $t=1$.

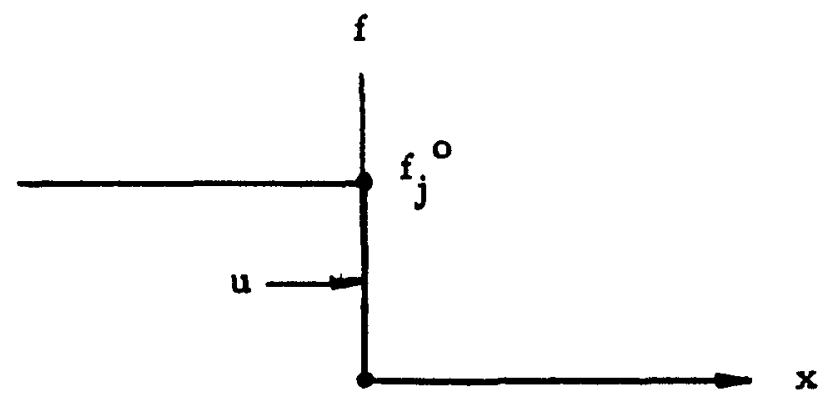

Fig. 23

$$
\text { Now, } f_{j}^{n+1}=f_{j}^{n}-\gamma\left(f_{j}^{n}-f_{j}-1^{n}\right)
$$

and for $Y=1 \quad f_{j}^{n+1}=f_{j-1}{ }^{n}$, or the fluid propagates in exactly the same condition as it was at $j=0$.

Actually in considering the stability we need only worry about small values of the wave length, aince long wave lengths spread over many grid pointe, giving smaller orrors. The error will be a maximum for $\frac{k \Delta x}{2}=\frac{\pi}{2}$

Physically $\frac{\Delta x}{\Delta t}$ defines the characteristic velocity of the grid and is the maximum velocity with which signals may be propagated through the grid. If $u>\frac{\Delta x}{\Delta t}$ the material cannot propagate acros the mesh at this velocity. This is also the condition imposed by stability on the mesh.

The above method works atisfactorily as far as memory storage is concerned, but is only accurato to first order. 
Second Order Differencing Scheme

A method which is satisfactory to second order will now be considered.

By expansion,

$$
\left.f(x, t+\Delta t)=f(x, t)+\Delta t\left(\frac{\partial f}{\partial t}\right)_{x, t}+\frac{(\Delta t)^{2}}{2} \frac{\theta^{2} f}{\partial t^{2}}\right]_{x, t}+\ldots
$$

But using the differential equation (71) we obtain

$$
\left.\left.f(x, t+\Delta t)=f(x, t)-u \Delta t \frac{\partial f}{\partial x}\right]_{t}+\frac{(\Delta t)^{2}}{2} u^{2} \frac{\partial^{2} f}{\partial x^{2}}\right]_{t} .
$$

Taking centered space differences,

$$
f_{j}^{n+1}=f_{j}^{n}-u \Delta t\left(\frac{f_{j+1}^{n}-f_{j}-1^{n}}{2 \Delta x}\right)+\frac{y^{2}}{2}\left(f_{j+1}{ }^{n}-2 f_{j}^{n}+f_{j}-1\right)
$$

which is centered at $(n, j)$, correct to second order, and independent of the oign of $u$.

If we apply the Von Noumann method to test for stability we arrive at the tability condition

$$
\gamma^{2} \leq 1
$$

This method appears to be satisfactory. It has been applied to the calculation of the progress of a contact discontinuity in Eulerian coordinates. The forward differencing method has also been applied to this calculation with results fllustrated in Fig. 24.

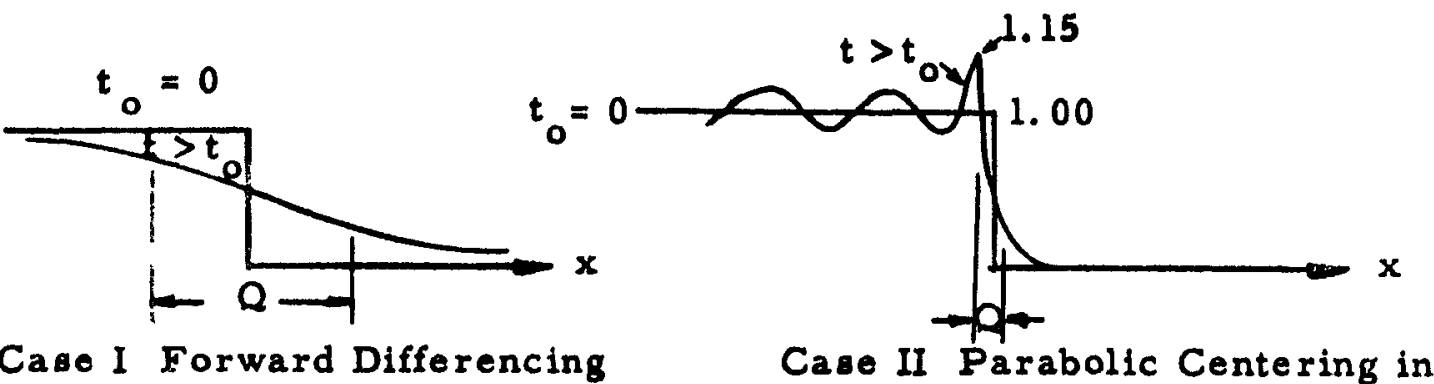


For case I the rate of growth of the "half width", $Q$, of the contact discontinuity was approximately proportional to $\sqrt{n_{1}}$ whereas in case II the rate of growth of $Q$ was slow, approximately as $\mathrm{n}^{0.1}$. In the latter case however there is an appreciable overshoot. A compromise combination of both methods has been used to yield a rate of growth varying as $n^{0.4}$ and with a three percent overshoot. 


\title{
UNIVERSITY OF CALIFORNIA, RADIATION LABORATORY LIVERMORE SITE
}

\section{LECTURES ON HYDRODYNAMICS AND SHOCK WAVES}

\author{
Lectures by Robert Lelevier - Notes by Robert Lelevier
}

May 14, 1954

Lecture 8: THE RICHTMEYER - VON NEUMANN Q-METHOD*

* J. Von Neumann and R. D. Richtmeyer, "A Method for the Numerical Calculation of Hydrodynamic Shocks", J. of Appl. Phys., 21, 232 - 237 (March 1950).

The presence of shocks can cause trouble in a numerical integration due to the discontinuities in density, entropy, pressure, etc. The partial differential equations require boundary conditions at the shock front as prescribed by the Hugoniot relations. However, if one is interested in doing an explicit stepwise integration in time, such knowledge of the boundary conditions is not available.

Richtmeyer and Von Neumann overcame this difficulty by exploiting the effect of dissipative mechanisms on shocks, namely, that of thickening the transition region. In other words, they replaced the mathematical discontinuity by a "rapid but continuous" transition. This was accomplished by the introduction of an artificial viscosity (in the form of a pressure, " $q$ ") in the equations of motion.

The idea works in the following fashion: wherever the flow is isentropic, since there are no discontinuities to deal with, $q$ is zero. How ever, if a shock begins to develop anywhere in the flow, then the qappears 
"utomatically and "rounds off" the discontinuity, thus keeping the numerical integration stable.*

* When one attempts to integrate the usual equations of motion using a convenient finite difference representation, the numerical results quickly become unrecognizable if the flow is such that shocks try to deve.op. It should be pointed out, however that the use of an artificial viscosity is not essential in handling a numerical integration. P. Lax of New York University has shown that by expressing all the equations of motion in "conservation" form, i. e., $\frac{\partial F(x, t)}{\partial t}+\frac{\partial G(x, t)}{\partial x}=0$, one can perform a successful numerical integration by a straightforward differencing of the partial differential equations even with the presence of shocks.

The equations of motion are changed to read as follows:

$$
\begin{aligned}
& \frac{D}{D t} \vec{u}+\frac{1}{\rho} \vec{\nabla}(p+q)=0 \\
& \frac{D}{D t} \rho+\rho \vec{\nabla} \cdot \vec{u}=0 \\
& \frac{D}{D t} \epsilon+(p+q) \frac{D}{D t} v=0 \quad\left(v=\frac{1}{\rho}\right) .
\end{aligned}
$$

If a shock is present, the term $q \frac{\mathrm{Dv}}{\overline{D t}}$ is to take care of the work done irreversibly across the shock. One must demand that:

(A) the equations possess solutions without discontinuities,

(B) the equations admit of a steady state solution where the thickness of the shock layer is independent of time and the strength of the shock, and

(C) the Hugoniot relations are obeyed in the large.

A nonlinear expression for $q$ was found which satisfied all the requirements, i.e.,

$$
q=\left\{\begin{aligned}
L^{2} \rho(\vec{\nabla} \cdot \vec{u})^{2} ; & \text { if } \vec{\nabla} \cdot \vec{u}<0 \\
0 & ; \text { if } \vec{\nabla} \cdot \vec{u} \geqq 0
\end{aligned}\right. \text {. }
$$


$L$ is a length, related to the spacial thickness of the net used in the numerical integration. The form for $q$ was chosen to be nonlinear in order to provide a sharp definition of the shock region.

We can investigate the properties of $q$ by considering a plane of discontinuity traveling with velocity $\vec{B}$. Let $\vec{n}$ be a unit vector normal to the plane. Let $s=\vec{B} \cdot \vec{n}$. (See Figure 25.)

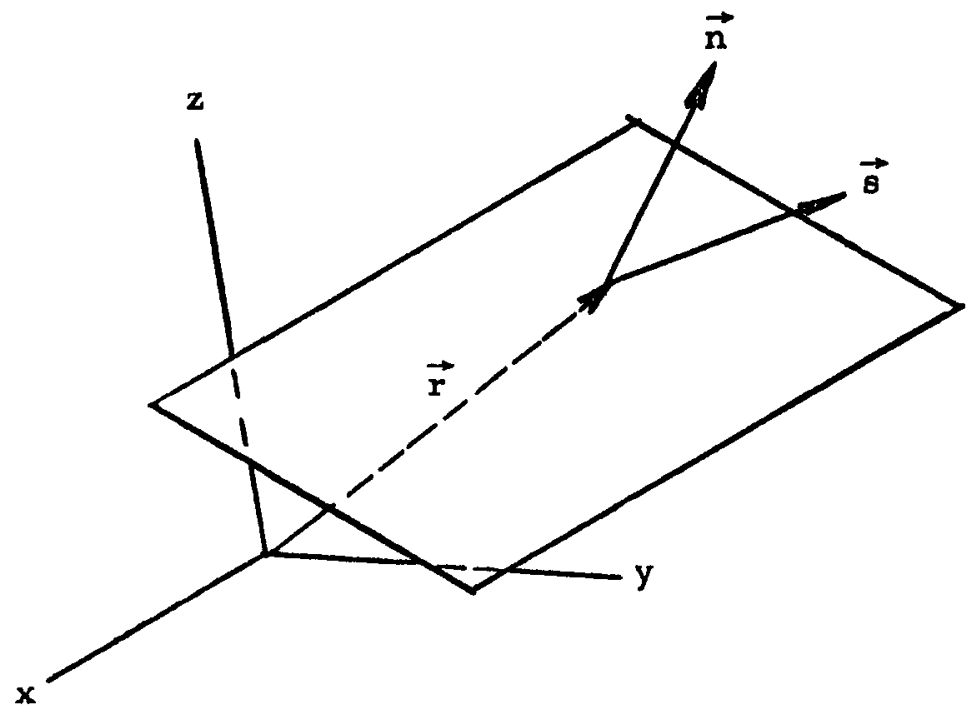

Fig. 25

In steady state, any function $f(x, y, z, t)$ can be expressed as

$$
\begin{aligned}
f & =f\left(\vec{n} \cdot\left[\vec{r}-\vec{s}^{t} t\right]\right) \\
& =f(\vec{n} \cdot \vec{r}-s t)
\end{aligned}
$$

Let $w=\vec{n} \cdot \vec{r}-8 t$ and let " $m$ " be the rate of mass flow across the discontinuity per unit area.

$$
m=\rho(\vec{u} \cdot \vec{n}-s)=\rho\left(u_{n}-s\right)=\text { constant for steady state. }
$$

The operator $\rho \frac{D}{D t}$ becomes

$$
\rho \frac{D}{D t}=\rho\left(\frac{\partial}{\partial t}+\vec{u} \cdot \vec{\nabla}\right)=\rho\left[-s+u_{n}\right] \frac{d}{d w}=m \frac{d}{d w} .
$$

Equations (92) (93) and (94) become 


$$
\begin{aligned}
& m \frac{d}{d w} \vec{u}+\vec{n} \frac{d}{d w}(p+q)=0 \\
& -m \frac{d v}{d w}+\frac{d}{d w} u_{n}=0 \\
& \frac{d \epsilon}{d w}+(p+q) \frac{d v}{d w}=0
\end{aligned}
$$

Equation (98) can be written, using (96) and (97), as

$$
m\left[\frac{d \epsilon}{d w}+\frac{d}{d w}\left(\frac{l}{2} \vec{u} \cdot \vec{u}\right)\right]+\frac{d}{d w}\left[(p+q) u_{n}\right]=0 .
$$

Equations (96) and (98a) can be integrated immediately and if we require that $\mathrm{q} \rightarrow 0$ as $\mathrm{w} \rightarrow \pm \infty$ then we arrive at the usual Rankine-Hugoniot relations:

$$
\begin{aligned}
& m\left(\vec{u}_{1}-\vec{u}_{2}\right)=-\vec{n}\left(p_{1}-p_{2}\right) \\
& m\left(v_{1}-v_{2}\right)=\vec{n} \cdot\left(u_{1}-u_{2}\right) \\
& m\left[\left(\frac{1}{2} \vec{u} \cdot \vec{u}\right)+\epsilon_{1}\right]-m\left[\left(\frac{1}{2} \vec{u} \cdot \vec{u}\right)+\epsilon_{2}\right]= \\
& -\left[p_{1}\left(\vec{u}_{1} \cdot \vec{n}\right)-p_{2}\left(\vec{u}_{2} \cdot \vec{n}\right)\right] .
\end{aligned}
$$

The subscript 1 refers to values ahead of the discontinuity $(w \rightarrow+\infty)$ and the subscript 2 refers to values behind the discontinuity $(w \rightarrow-\infty)$.

If we multiply equation (99) by (n.) we obtain

$$
p_{1}-p_{2}=-m\left(u_{1 n}-u_{2 n}\right)
$$

Let $\vec{\lambda}$ be a unit vector in the plane of the discontinuity. $(\vec{\lambda} \cdot \vec{n}=0)$. Multiply equation (99) by $\vec{\lambda}$ :

$$
m \vec{\lambda} \cdot\left(\vec{u}_{1}-\vec{u}_{2}\right)=m\left(u_{1 t}-u_{2 t}\right)=0 \text {. }
$$

Discontinuities for which $m=0$ are called slip streams. From (102) it follows that the pressure is continuous across a slip stream. From (100) we see that the normal components of the material velocity are also continuous across a slip stream. However from (10,3) we see that the tangential components are not necessarily continuous.

If $\mathrm{m} \neq 0$, we have a shock. 
So far we see that if $q \rightarrow 0$ as $w \rightarrow \pm$ co (that is, $(\vec{r} \cdot \vec{n}-s t)$ is large compared to some fundamental length associated with the grid spacings) then condition (C) is satisfied.

To examine condition (B) write q as

$$
q=L^{2} \frac{1}{v^{3}}\left(\frac{D v}{D t}\right)^{2}, u \operatorname{sing} \vec{\nabla} \cdot \vec{u}=\frac{1}{v} \underset{D t}{D}
$$

or

$$
q v=L^{2} m^{2}\left(\frac{d v}{d w}\right)^{2}
$$

From equations (96) to (98) we obtain, using a $\gamma$-law equation of state,

$$
q v=-\frac{y+1}{2} m^{2} v^{2}+c_{0}+c_{1} v
$$

where $c_{0}$ and $c_{1}$ are constants of integration. Equation (105) is quadratic in $v$ and we require that $q=0$ when $v=v_{1}$ or $v=v_{2}$. By suitably choosing the constants of integration we can rewrite (105):

$$
q v=\frac{y+1}{2} m^{2}\left(v_{1}-v\right)\left(v-v_{2}\right)
$$

Hence (104) and (106) give the following differential equation for $v$ through the tranaition region:

$$
L^{2}\left(\frac{d v}{d w}\right)^{2}=\frac{y+1}{2}\left(v_{1}-v\right)\left(v-v_{2}\right) .
$$

Thio can be integrated giving

where

$$
\begin{aligned}
& v=\frac{v_{1}+v_{2}}{2}+\frac{v_{1}-v_{2}}{2} \sin \frac{w}{w_{0}} \\
& w_{0}=L \sqrt{\frac{2}{\gamma+1}}
\end{aligned}
$$

Thus the transition region has a thickness $\pi L \sqrt{\frac{2}{\gamma+1}}$.

Rixhtmeyer and Von Neumann define $q$ essentially as follows:

$$
q=(c \Delta x)^{2} \frac{1}{v^{3}}\left(\frac{d v}{d t}\right)^{2}
$$


Hence to spread the shock out over $\mathrm{N}$ zones,

$$
L=\frac{N \Delta x}{\pi} \sqrt{\frac{Y+1}{2}} \text { or } \quad c=\frac{N}{\pi} \sqrt{\frac{Y+1}{2}}
$$

For problems in two-dimensional flow, where $\Delta x$ is not necessarily equal to $\Delta y$, then the choice of the value to use for $L$ depends on the direction of propagation of the shock. If $\vec{n}$ is a unit vector normal to the shock front, then in the expression for $L, \Delta x$ can be replaced by

$$
\frac{\Delta x \Delta y}{\left[(\Delta y \cos \theta)^{2}+(\Delta x \sin \theta)^{2}\right]^{1 / 2}}
$$

where $\Delta x$ and $\Delta y$ are the grid spacings in the $x$ and $y$ directions and $\theta$ is the inclination of the normal with respect to the $x$ axis.

In Equation (108) it should be noted that for a fixed $N$, the constant in $q$ depends on the equation of state, $i . e .$, on $\gamma$, of the material through which the shock is running. 
DISTRIBUTION

No. of Copies

LRL Internal Distribution,

Information Division

George Bing

Eugene Goldberg

Stuart Stone

Robert Remillard

Ervin Woodward

Louis Wouters

Charles Godfrey

James Frank

James Tracy

Forrest Fairbrother

K. Bandtel

David Oakley

Donald Born

Mel Harrison

Richard Werner

William L. Barr

LRL Berkeley,

R. K. Wakerling, Technical Information

External Distribution,

Division of Technical Information Extension, Oak Ridge 